\title{
Adaptive synchronization of stochastic complex dynamical networks and its application
}

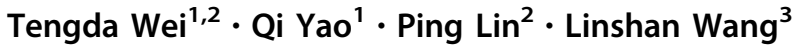

Received: 20 December 2017 / Accepted: 20 April 2018/Published online: 9 May 2018

(C) The Author(s) 2018

\begin{abstract}
This paper investigates exponential synchronization for stochastic complex dynamical networks with reaction-diffusion terms and S-type distributed delays. Based on a generalized Halanay inequality and Poincaré inequality, adaptive control strategies for exponential synchronization are established by constructing a simple Lyapunov-Krasovskii functional candidate and utilizing the truncation method. Some numerical examples are provided to demonstrate the effectiveness of the obtained results. Finally, the proposed adaptive synchronization theoretical results are successfully applied to image encryption.
\end{abstract}

Keywords Adaptive synchronization - Stochastic complex dynamical network - S-type distributed delay · Reaction-diffusion · Image encryption

Mathematics Subject Classification 34D06 $\cdot 90 \mathrm{~B} 15 \cdot 34 \mathrm{~K} 50 \cdot 68 \mathrm{U} 10$

\section{Introduction}

Complex dynamical networks widely exist in our life, such as communication networks, social networks, power grids, cellular networks, and secure communication. The behavior of complex dynamical networks has good explanation of real-world phenomena [15, 22]. Therefore, many researchers have intensively studied the dynamical behavior and topologies of complex dynamical networks $[3,5,12,25,32,36]$. In particular, synchronization is

Linshan Wang

wangls@ouc.edu.cn

Tengda Wei

tdwei123@163.com

Qi Yao

qiyao8@126.com

Ping Lin

plin@maths.dundee.ac.uk

1 College of Oceanic and Atmospheric Sciences, Ocean University of China, Qingdao 266100, China

2 Department of Mathematics, University of Dundee, Dundee DD1 4HN, UK

3 School of Mathematical Sciences, Ocean University of China, Qingdao 266100, China undoubtedly the important collective behavior because of its mathematical importance and potential applications $[21,24]$. The synchronized nodes share the same patterns by some control strategies, such as feedback control $[10,38]$, impulsive control [2, 37], sample-data control $[17,34]$, and adaptive control $[1,11]$.

In realistic world, stochastic perturbations are unavoidable in the propagation of electric potential in a neuron and brings essential change to the neural networks [46]. Generally speaking, two types of stochastic perturbations are introduced into the analysis of stochastic complex dynamical networks (SCDNs): Markovian jumping parameters and finite dimensional Brownian motions, both of which have attracted much attention of researchers $[18,19]$. Proper control strategies are derived to synchronize the state trajectory of SCDNs [8, 30, 35]. Recently, the diffusion phenomena are also considered in SCDNs because the electrons sometimes move in nonhomogeneous field. The state variables of SCDNs with reaction-diffusion terms are related to both time and space such that the synchronization criteria should be established in Hilbert space instead of Euclidean Space [13]. Hence, it is necessary to investigate the synchronization of SCDNs with reaction-diffusion terms $[2,4,7,29,37]$. 
On the other hand, image encryption has attracted intensive attention [41-44] because the security of confidential digital images is very important in public transmission. In recent years, many researchers have focused on chaos-based encryption algorithms which rely on the dynamics of the systems [40, 45]. For instance, Zhang and Wang [45] investigated a new spatiotemporal dynamics with fractional order differential logistic map and spatial nonlinear coupling, which are more suitable for encryptions than the former adjacent coupled map lattices. Synchronization of delayed chaotic system in secure communication was also studied in [4, 20, 33]. In [4], Chen et al. presented a new impulsive synchronization criterion of two identical reaction-diffusion neural networks with discrete and unbounded distributed delays. The developed impulsive synchronization method is applied to build a spatiotemporal chaotic cryptosystem. Further development about application of delayed neural networks to image encryption refers to $[4,9]$ and references therein. S-type distributed delay not only includes discrete time delay and distributed time delay but also has better description of hysteresis phenomena in networks, and stochastic delayed system may have promising application to image encryption. But there is relatively less work on synchronization and application of SCDNs with reaction-diffusion terms and S-type distributed delays.

Motivated by above discussion, we study SCDNs with reaction-diffusion terms and S-type distributed delays, aiming to develop adaptive strategies for synchronization and apply the theoretical results to image encryption. The novelty of our contribution lies in the following aspects: (1) Adaptive synchronization is investigated for SCDNs with reaction-diffusion terms and S-type distributed delays which are infinite delays; (2) the S-type distributed delays are handled by a novel generalized Halanay inequality and the truncation method so that the used Lyapunov-Krasovskii functional candidate is a sample one without distributed term; (3) the synchronization criteria are established in Hilbert space due to the existence of reaction-diffusion terms; (4) the adaptive synchronization results are successfully applied to image encryption based on a spatiotemporal cryptosystem proposed in [4].

Notations $\mathbb{H}=\left(H_{0}^{1}(\mathbb{O})\right)^{n}$ and $\mathbb{L}=\left(L^{2}(\mathbb{O})\right)^{n}$ are Hilbert spaces with norms $\|\boldsymbol{u}\|_{\mathbb{H}}=\left(\sum_{i=1}^{n} \sum_{j=1}^{l} \int_{\mathbb{O}}\left(\frac{\partial u_{i}}{\partial x_{j}}\right)^{2} \mathrm{~d} \boldsymbol{x}\right)^{\frac{1}{2}}$ and $\|\boldsymbol{u}\|_{\mathbb{L}}=\left(\sum_{i=1}^{n} \int_{\mathbb{O}}\left|u_{i}\right|^{2} \mathrm{~d} \boldsymbol{x}\right)^{\frac{1}{2}}$. $(\cdot, \cdot)$ is the inner product of $\mathbb{L}$. $(\Omega, \mathcal{F}, P)$ is a complete probability space with filtration $\left\{\mathcal{F}_{t}\right\}_{t \geq 0} . \mathbb{C}=C((-\infty, 0], \mathbb{L})$ represents the Banach space of all continuous functions from $(-\infty, 0]$ to $\mathbb{L}$ with norm $\|\boldsymbol{\varphi}\|_{\mathbb{C}}=\sup _{\theta \leq 0}\|\boldsymbol{\varphi}(\theta)\| . \mathscr{L}\left(\left(L^{2}(\mathbb{O})\right)^{m}, \mathbb{L}\right)$ represents the space of all linear bounded operators from $\left(L^{2}(\mathbb{O})\right)^{m}$ into $\mathbb{L}$ with operator norm. $\|\boldsymbol{A}\|_{F} \triangleq\left(\operatorname{tr}\left(\boldsymbol{A} \boldsymbol{A}^{T}\right)\right)^{\frac{1}{2}}$ is the Frobenius norm of $\boldsymbol{A} \in \mathbb{R}^{n \times n}$, and $t r$ is the trace operator. For any continuous $\mathcal{F}_{t}$-adapted $\mathbb{\text { -valued }}$ stochastic process $\boldsymbol{u}(t, \boldsymbol{x})(\omega): \Omega \rightarrow \mathbb{L}$, we define a continuous $\mathcal{F}_{t}$-adapted $\mathbb{C}$ valued stochastic process $\boldsymbol{u}_{t}(\omega)=\boldsymbol{u}(t+\theta, \boldsymbol{x})(\omega)$ and $\left\|\boldsymbol{u}_{t}\right\|_{\mathbb{C}}=\sup _{\theta \leq 0} \mathcal{E}\|\boldsymbol{u}(t+\theta)\|, \omega \in \Omega, \boldsymbol{x} \in \mathbb{O}, t \geq 0$ where $\mathcal{E}(\cdot)$ is the expectation operator. $\mathbb{C}_{\mathcal{F}_{0}}^{b}$ denotes the family of $\mathcal{F}_{0}$-measurable bounded $C\left((-\infty, 0], \mathbb{R}^{n}\right)$-valued stochastic variables $\phi$ with $\mathcal{E}\|\boldsymbol{\phi}\|_{\mathbb{C}}<\infty . L_{\mathcal{F}_{t}}(\Omega, \mathbb{L})$ is the family of $\mathbb{L}$ valued $\mathcal{F}_{t}$-measurable random variables $\xi$ with $\mathcal{E}\|\xi\|^{2}<\infty$.

\section{Problem formulation and preliminaries}

In this paper, we consider the following SCDNs with reaction-diffusion terms and S-type distributed delays

$$
\begin{aligned}
& \mathrm{d} \boldsymbol{s}(t, \boldsymbol{x})=\left[\nabla \cdot(\boldsymbol{D}(\boldsymbol{x}) \circ \nabla \boldsymbol{s}(t, \boldsymbol{x}))-\boldsymbol{A}\left(\gamma_{t}\right) \boldsymbol{s}(t, \boldsymbol{x})\right. \\
& +\boldsymbol{B}_{1}\left(\gamma_{t}\right) \boldsymbol{f}(\boldsymbol{s}(t, \boldsymbol{x}))+\boldsymbol{I}(t)+\boldsymbol{B}_{2}\left(\gamma_{t}\right) \boldsymbol{f} \\
& \left.\left(\int_{-\infty}^{0} \mathrm{~d} \boldsymbol{\eta}(\theta) \boldsymbol{s}(t+\theta, \boldsymbol{x})\right)\right] \mathrm{d} t+\boldsymbol{g}\left(\boldsymbol{s}, \int_{-\infty}^{0} \mathrm{~d} \boldsymbol{\eta}(\theta) \boldsymbol{s}(t+\theta, \boldsymbol{x})\right) \mathrm{d} \boldsymbol{w}(t),
\end{aligned}
$$

where $t \geq 0, \boldsymbol{x} \in \mathbb{O}, \boldsymbol{s}(t, \boldsymbol{x})=\left(s_{1}(t, \boldsymbol{x}), s_{2}(t, \boldsymbol{x}), \cdots, s_{n}(t, \boldsymbol{x})\right)^{T}$, $\nabla \cdot(\boldsymbol{D}(\boldsymbol{x}) \circ \nabla \boldsymbol{s})=\left(\sum_{j=1}^{l} \frac{\partial\left(D_{1 / j x_{j}}\right)}{\partial x_{j}}, \cdots, \sum_{j=1}^{l} \frac{\partial\left(D_{j j} \frac{\partial s_{n}}{\partial x_{j}}\right)}{\partial x_{j}}\right)^{T}, \boldsymbol{D}(\boldsymbol{x})=$ $\left(D_{i j}(\boldsymbol{x})\right)_{n \times l}, \quad D_{i j}(\boldsymbol{x}) \geq \hat{D}_{i j}>0$, and $\circ$ denotes Hadamard product [13]. $\int_{-\infty}^{0} \mathrm{~d} \boldsymbol{\eta}(\theta) \boldsymbol{s}(t+\theta, \boldsymbol{x})$ is Lebesgue-Stieltjes integrable, and $\eta_{i j}(\theta)(i, j=1,2, \ldots, n)$ is non-decreasing bounded variation function which satisfies $\int_{-\infty}^{0} d \eta_{i j}(\theta)=\hat{\eta}_{i j}>0 . \boldsymbol{I}(t)=\left(I_{1}(t), \ldots, I_{n}(t)\right)^{T}$ is an external input. $\mathbb{O}$ is an open bounded and connected subset of $\mathbb{R}^{l}$ with a sufficient regular boundary $\partial \mathbb{O}$. $\boldsymbol{w}(t)$ is a Brownian motion defined on the complete probability space and $\boldsymbol{g} \in M_{2}^{n, m}(0, t)$.

$\left\{\gamma_{t}, t \geq 0\right\}$ is a right-continuous Markov process on the probability space which takes values in the finite space $\mathbb{M}=\{1,2, \ldots, M\}$ with jumping transfer matrix $\Pi=$ $\left\{\pi_{m n}\right\}(m, n \in \mathbb{M})$ given by

$$
P\left\{\gamma_{t+\Delta t}=n \mid \gamma_{t}=m\right\}= \begin{cases}\pi_{m n} \Delta t+o(\Delta t), & \text { if } \mathrm{m} \neq \mathrm{n} ; \\ 1+\pi_{m n} \Delta t+o(\Delta t), & \text { if } \mathrm{m}=\mathrm{n},\end{cases}
$$

where $\Delta t>0$ and $\lim _{\Delta t \rightarrow 0} o(\Delta t) / \Delta t=0, \pi_{m n} \geq 0$ is the transition rate from $m$ to $n$ if $m \neq n$ and $\pi_{m m}=-\sum_{n \neq m} \pi_{m n}$. The Brownian motion is independent of Markov process. The matrices $\boldsymbol{A}\left(\gamma_{t}\right), \boldsymbol{B}_{1}\left(\gamma_{t}\right)$ and $\boldsymbol{B}_{2}\left(\gamma_{t}\right)$ are real-valued Markovian jumping parameters. For the sake of simplicity, we denote $\boldsymbol{A}\left(\gamma_{t}\right), \boldsymbol{B}_{1}\left(\gamma_{t}\right), \boldsymbol{B}_{2}\left(\gamma_{t}\right)$ by $\boldsymbol{A}_{m}$, $\boldsymbol{B}_{1 m}, \boldsymbol{B}_{2 m}$ for $\gamma_{t}=m \in \mathbb{M}$. Then, when $\gamma_{t}=m$, the above SCDNs can be rewritten as 
$\mathrm{d} \boldsymbol{s}(t, \boldsymbol{x})=\left[\nabla \cdot(\boldsymbol{D}(\boldsymbol{x}) \circ \nabla \boldsymbol{s}(t, \boldsymbol{x}))-\boldsymbol{A}_{m} \boldsymbol{s}(t, \boldsymbol{x})+\boldsymbol{B}_{1 m} \boldsymbol{f}(\boldsymbol{s}(t, \boldsymbol{x}))\right.$

$\left.+\boldsymbol{B}_{2 m} \boldsymbol{f}(\mathcal{S}(\boldsymbol{s}))+\boldsymbol{I}(t)\right] \mathrm{d} t+\boldsymbol{g}(\boldsymbol{s}, \mathcal{S}(\boldsymbol{s})) \mathrm{d} \boldsymbol{w}(t)$,

where $\mathcal{S}(\boldsymbol{s})=\int_{-\infty}^{0} \mathrm{~d} \boldsymbol{\eta}(\theta) \boldsymbol{s}(t+\theta, \boldsymbol{x})$. We consider neural network (2) as drive system. The response SCDNs consisting of $N$ linearly coupled identical nodes are described as

$$
\begin{aligned}
\mathrm{d} \boldsymbol{u}_{i}(t, \boldsymbol{x})= & {\left[\nabla \cdot\left(\boldsymbol{D}(\boldsymbol{x}) \circ \nabla \boldsymbol{u}_{i}(t, \boldsymbol{x})\right)-\boldsymbol{A}_{m} \boldsymbol{u}_{i}(t, \boldsymbol{x})\right.} \\
& +\boldsymbol{B}_{1 m} \boldsymbol{f}\left(\boldsymbol{u}_{i}(t, \boldsymbol{x})\right)+\boldsymbol{B}_{2 m} \boldsymbol{f}\left(\mathcal{S}\left(\boldsymbol{u}_{i}\right)\right) \\
& +\sum_{j=1}^{N} c_{i j} \boldsymbol{G}_{m}\left(\boldsymbol{u}_{j}+\mathcal{S}\left(\boldsymbol{u}_{j}\right)\right) \\
& \left.+\boldsymbol{U}_{i}(t, \boldsymbol{x})+\boldsymbol{I}(t)\right] \mathrm{d} t+\boldsymbol{g}\left(\boldsymbol{u}_{i}, \mathcal{S}\left(\boldsymbol{u}_{i}\right)\right) \mathrm{d} \boldsymbol{w}(t),
\end{aligned}
$$

where $\quad \boldsymbol{u}_{i}(t, \boldsymbol{x})=\left(u_{i 1}(t, \boldsymbol{x}), u_{i 2}(t, \boldsymbol{x}), \ldots, u_{i n}(t, \boldsymbol{x})\right)^{T} \quad$ and $i=1,2, \ldots, N, m \in \mathbb{M}$. $\boldsymbol{U}_{i}(t, \boldsymbol{x})$ is the control input. $\boldsymbol{G}_{m}$ is the inner coupling positive definite matrix, and $\left(c_{i j}\right)_{N \times N}$ is the coupling matrix which satisfies $c_{i i}=-\sum_{j \neq i} c_{i j}$ and $c_{i j}>0$ if node $j$ is connected to node $i$, otherwise, $c_{i j}=0$. Defining $\boldsymbol{v}_{i}(t, \boldsymbol{x})=\boldsymbol{u}_{i}(t, \boldsymbol{x})-\boldsymbol{s}(t, \boldsymbol{x})$ as the synchronization error of the drive system (2) and response system (3), the error system with initial condition and Dirichlet boundary condition can be expressed by

$$
\left\{\begin{aligned}
& \mathrm{d} \boldsymbol{v}_{i}(t, \boldsymbol{x})=[\nabla \cdot\left(\boldsymbol{D}(\boldsymbol{x}) \circ \nabla \boldsymbol{v}_{i}(t, \boldsymbol{x})\right)-\boldsymbol{A}_{m} \boldsymbol{v}_{i}(t, \boldsymbol{x})+\boldsymbol{B}_{1 m} \boldsymbol{F}\left(\boldsymbol{v}_{i}(t, \boldsymbol{x})\right) \\
&\left.+\boldsymbol{B}_{2 m} \boldsymbol{F}\left(\mathcal{S}\left(\boldsymbol{v}_{i}\right)\right)+\sum_{j=1}^{N} c_{i j} \boldsymbol{G}_{m}\left(\boldsymbol{v}_{j}+\mathcal{S}\left(\boldsymbol{v}_{j}\right)\right)+\boldsymbol{U}_{i}(t, \boldsymbol{x})\right] \mathrm{d} t \\
&+\boldsymbol{\sigma}\left(\boldsymbol{v}_{i}, \mathcal{S}\left(\boldsymbol{v}_{i}\right)\right) \mathrm{d} \boldsymbol{w}(t), t \geq 0, \boldsymbol{x} \in \mathbb{O} \\
&\left.\boldsymbol{v}_{i}(t, \boldsymbol{x})\right|_{x \in \hat{0} \mathbb{O}}=0, t \geq 0 \\
& \boldsymbol{v}_{i}(\theta, \boldsymbol{x})=\boldsymbol{\phi}_{i}(\theta, \boldsymbol{x}), \theta \in(-\infty, 0], \boldsymbol{x} \in \mathbb{O}
\end{aligned}\right.
$$

where

$$
\begin{aligned}
& \boldsymbol{F}\left(\boldsymbol{v}_{i}\right)=\boldsymbol{f}\left(\boldsymbol{u}_{i}(t, \boldsymbol{x})\right)-\boldsymbol{f}(\boldsymbol{s}(t, \boldsymbol{x})), \\
& \boldsymbol{F}\left(\mathcal{S}\left(\boldsymbol{v}_{i}\right)\right)=\boldsymbol{f}\left(\mathcal{S}\left(\boldsymbol{u}_{i}\right)\right)-\boldsymbol{f}(\mathcal{S}(\boldsymbol{s})), \\
& \boldsymbol{\sigma}\left(\boldsymbol{v}_{i}, \mathcal{S}\left(\boldsymbol{v}_{i}\right)\right)=\boldsymbol{g}\left(\boldsymbol{u}_{i}, \mathcal{S}\left(\boldsymbol{u}_{i}\right)\right)-\boldsymbol{g}(\boldsymbol{s}, \mathcal{S}(\boldsymbol{s})) .
\end{aligned}
$$

Then, we list the definitions and lemmas which will be used throughout the paper.

Definition 1 [16] The drive system (2) and response system (3) are globally exponentially synchronized in mean square, if there exist positive constants $M$ and $\lambda$, such that

$$
\mathcal{E} \sum_{i=1}^{N}\left\|\boldsymbol{v}_{i}(t, \boldsymbol{x})\right\|^{2} \leq M e^{-\lambda t} \mathcal{E} \sum_{i=1}^{N}\left\|\boldsymbol{\phi}_{i}\right\|_{\mathbb{C}}^{2} .
$$

Definition 2 [28] Let $\quad \tilde{\mathbb{C}}=C\left([t-\tau, t], \mathbb{R}^{n}\right), \quad \tau \geq 0$, $\boldsymbol{\Psi}(t, \boldsymbol{x}, \boldsymbol{y}) \in C\left(\mathbb{R}^{+} \times \mathbb{R}^{n} \times \tilde{\mathbb{C}}, \mathbb{R}^{n}\right) . \quad \boldsymbol{\Psi}(t, \boldsymbol{x}, \boldsymbol{y})=\left(\Psi_{1}, \Psi_{2}\right.$, $\left.\ldots, \Psi_{n}\right)^{T}$ is called an M-function, if (i) for every $t \in \mathbb{R}^{+}, \boldsymbol{x} \in \mathbb{R}^{n}, \boldsymbol{y}^{(1)}, \boldsymbol{y}^{(2)} \in \tilde{\mathbb{C}}$,

$$
\boldsymbol{\Psi}\left(t, \boldsymbol{x}, \boldsymbol{y}^{(1)}\right) \leq \boldsymbol{\Psi}\left(t, \boldsymbol{x}, \boldsymbol{y}^{(2)}\right) \text {, for } \boldsymbol{y}^{(1)} \leq \boldsymbol{y}^{(2)},
$$

where $\quad \boldsymbol{y}^{(1)}=\left(y_{1}^{(1)}, \ldots, y_{n}^{(1)}\right)^{T}, \quad \boldsymbol{y}^{(2)}=\left(y_{1}^{(2)}, \ldots\right.$, $\left.y_{n}^{(2)}\right)^{T}$

(ii) every ith element of $\boldsymbol{\Psi}((t, \boldsymbol{x}, \boldsymbol{y})$ satisfies

$\Psi_{i}\left(t, \boldsymbol{x}^{(1)}, \boldsymbol{y}\right) \leq \Psi_{i}\left(t, \boldsymbol{x}^{(2)}, \boldsymbol{y}\right)$, for any $\boldsymbol{y} \in \tilde{\mathbb{C}}, t \geq t_{0}$,

where arbitrary $\boldsymbol{x}^{(1)} \leq \boldsymbol{x}^{(2)}$ belonging to $\mathbb{R}^{n}$ and having the same ith component $x_{i}^{(1)}=x_{i}^{(2)}, \boldsymbol{x}^{(1)}=$ $\left(x_{1}^{(1)}, x_{2}^{(1)}, \ldots, x_{n}^{(1)}\right)^{T}, \boldsymbol{x}^{(2)}=\left(x_{1}^{(2)}, x_{2}^{(2)}, \ldots, x_{n}^{(2)}\right)^{T}$.

Lemma 1 (Poincaré inequality [23]) Let $\mathbb{O}$ be an open bounded smooth domain in $\mathbb{R}^{l}$, then

$\|\boldsymbol{u}\| \leq \alpha^{-1}\|\boldsymbol{u}\|_{\mathbb{H}}, \boldsymbol{u} \in \mathbb{H}$,

where the constant $\alpha>0$ depends on the size of the domain $\mathbb{O}$.

Lemma 2 [31] Let $\boldsymbol{x} \in \mathbb{R}^{n}, \boldsymbol{y} \in \mathbb{R}^{n}$ and $\rho>0$. Then, we have $\boldsymbol{x}^{T} \boldsymbol{y}+\boldsymbol{y}^{T} \boldsymbol{x} \leq \rho \boldsymbol{x}^{T} \boldsymbol{x}+\rho^{-1} \boldsymbol{y}^{T} \boldsymbol{y}$.

Lemma 3 [47] Assume that $\boldsymbol{A}, \boldsymbol{B}$ are $N \times N$ Hermitian matrices. Suppose that $\lambda_{1} \geq \lambda_{2} \geq \cdots \geq \lambda_{N}, \quad \mu_{1} \geq \mu_{2}$ $\geq \cdots \geq \mu_{N}$ and $\rho_{1} \geq \rho_{2} \geq \cdots \geq \rho_{N}$ are the eigenvalues of $\boldsymbol{A}, \boldsymbol{B}$ and $\boldsymbol{A}+\boldsymbol{B}$, respectively. Then, one has $\lambda_{i}+\mu_{N}$ $\leq \rho_{i} \leq \lambda_{i}+\mu_{1}, i=1,2, \ldots, N$.

Lemma 4 [27] Let $\boldsymbol{x}(t)=\left(x_{1}(t), \ldots, x_{n}(t)\right)^{T}, \quad \boldsymbol{y}(t)=$ $\left(y_{1}(t), \ldots, y_{n}(t)\right)^{T}, \overline{\boldsymbol{x}}(t)=\sup _{-\tau \leq \theta \leq 0} \boldsymbol{x}(t+\theta)$, and $\overline{\boldsymbol{y}}(t)=$ $\sup _{-\tau \leq \theta \leq 0} \boldsymbol{y}(t+\theta)$. Then, $\boldsymbol{x}(t)<\boldsymbol{y}(t)$ for $t \geq t_{0}$, if the following conditions
$\left(L_{1}\right) \boldsymbol{x}(t)<\boldsymbol{y}(t), t \in\left[t_{0}-\tau, t_{0}\right]$
$\left(L_{2}\right) D^{+} \boldsymbol{y}(t)>\boldsymbol{\Psi}(t, \boldsymbol{y}(t), \overline{\boldsymbol{y}}(t)), t \geq t_{0} \geq 0$
$\left(L_{3}\right) D^{+} \boldsymbol{x}(t) \leq \boldsymbol{\Psi}(t, \boldsymbol{x}(t), \overline{\boldsymbol{x}}(t)), t \geq t_{0} \geq 0$,

hold, where $\boldsymbol{\Psi}(t, \boldsymbol{x}, \boldsymbol{y})$ is an M-function.

Throughout the paper, we assume that the neuron activation functions satisfy the following condition: $\| f\left(\varsigma_{1}\right)-$ $\boldsymbol{f}\left(\varsigma_{2}\right)\left\|^{2} \leq L^{2}\right\| \varsigma_{1}-\varsigma_{2} \|^{2}$ and $\left\|\boldsymbol{g}\left(\varsigma_{1}, \varsigma_{2}\right)-\boldsymbol{g}\left(\hat{\boldsymbol{\varsigma}}_{1}, \hat{\boldsymbol{\varsigma}}_{2}\right)\right\|^{2} \leq L^{2}$ $\left(\left\|\varsigma_{1}-\hat{\varsigma}_{1}\right\|^{2}+\left\|\varsigma_{2}-\hat{\varsigma}_{2}\right\|^{2}\right)$, where $\varsigma_{1}, \varsigma_{2}, \hat{\varsigma}_{1}, \hat{\varsigma}_{2} \in \mathbb{R}^{n}$.

\section{Main results}

In this section, we will derive adaptive strategy and adaptive pinning strategy for exponential synchronization of SCDNs with reaction-diffusion terms and S-type distributed delays. First, the following generalized Halanay inequality is introduced to establish the sufficient conditions of adaptive synchronization. 
Lemma 5 (Generalized Halanay Inequality) If $\Phi(t) \geq 0$ satisfies

(i) $\Phi(t) \leq M, t \in(-\infty, 0]$;

(ii) for any $\epsilon \geq 0$, there exists $\tau \geq 0$ such that $\frac{\mathrm{d} \Phi(t)}{\mathrm{d} t} \leq-a \Phi(t)+b \sup _{-\tau \leq \theta \leq 0} \Phi(t+\theta)+o(\epsilon), t \in[0,+\infty)$,

where $\quad b>0, \quad a-b>0, \quad o(\epsilon) \geq 0, \quad$ and $\lim _{\epsilon \rightarrow 0} o(\epsilon)=0$, then there exists a positive constant $\lambda$ such that $\Phi(t) \leq M e^{-\lambda t}$ for $t \in[0,+\infty)$.

Proof For $\tau \in[0,+\infty)$, let us consider the following function

$\delta(\lambda)=-\lambda+a-b e^{\lambda \tau}$

where $\lambda \in[0,+\infty)$. Since $a-b>0$ and $b>0$, we obtain $\delta(0)>0$ and $\delta(\lambda)$ is continuous and monotonous, furthermore, $\delta(\lambda) \rightarrow-\infty$ as $\lambda \rightarrow+\infty$. Thus, there exists a constant $\lambda_{0}>0$ such that $-\lambda_{0}+a-b e^{\lambda_{0} \tau}>0$. Let $\Psi(t, x, y)=-a x+b y+o(\epsilon)$ and $y(t)=M e^{-\lambda_{0} t}+o(\epsilon) /$ $(a-b)$. From Definition 2, we know that $\Psi(t, x, y)$ is an M-function. Obviously, when $t \in(-\infty, 0], \quad \Phi(t) \leq M<$ $y(t)$. For $t \geq 0$, we have

$$
\begin{aligned}
D^{+} \Phi(t) & \leq-a \Phi(t)+b \sup _{-\tau \leq \theta \leq 0} \Phi(t+\theta)+o(\epsilon)=\Psi(t, \Phi(t), \bar{\Phi}(t)), \\
D^{+} y(t) & =-\lambda_{0} M e^{-\lambda_{0} t}>\left(-a+b e^{\lambda_{0} \tau}\right) M e^{-\lambda_{0} t} \\
& \geq-a y(t)+b \sup _{-\tau \leq \theta \leq 0} y(t+\theta)+o(\epsilon)=\Psi(t, y(t), \bar{y}(t)) .
\end{aligned}
$$

From Lemma 4, we know that $\Phi(t) \leq M e^{-\lambda_{0} t}+o(\epsilon) /$ $(a-b)$. As $\epsilon \rightarrow 0$, we obtain that $\Phi(t) \leq M e^{-\lambda_{0} t}$ for $t \in[0,+\infty)$.

Remark 1 Lemma 5 generalizes the classical Halanay inequality [6] to inequality defined on $(-\infty,+\infty)$. By employing Lyapunov method and Lemma 5, dynamical behavior (such as stability and synchronization) for a class of systems with infinite delays can be analyzed.

Adaptive strategy is a classical method to tune driveresponse systems toward reaching synchronization. The adaptive controller can be expressed by

$\boldsymbol{U}_{i}(t, \boldsymbol{x})=-k_{i}(t) \boldsymbol{v}_{i}(t, \boldsymbol{x}), i=1,2, \ldots, N$,

where $k_{i}(t)$ is the adaptive coupling strength.

Theorem 1 The drive system (2) and response system (3) are exponentially synchronous in mean square when the adaptive law is designed as $\dot{k}_{i}(t)=\rho_{i}\left\|\boldsymbol{v}_{i}(t, \boldsymbol{x})\right\|^{2}$ $(i=1,2, \ldots, N)$ where $\rho_{i}$ is an arbitrary positive constant.
Proof Given $\boldsymbol{\phi}_{i} \in \mathbb{C}_{\mathcal{F}_{0}}^{b}$ and fixed system mode $m \in \mathbb{M}$ arbitrarily, we write $\boldsymbol{v}_{i}(t, \boldsymbol{x})=\boldsymbol{v}_{i}\left(t, \boldsymbol{x} ; \boldsymbol{\phi}_{i}\right)$ and define a Lyapunov-Krasovskii functional candidate by

$$
\begin{aligned}
& V\left(t, \boldsymbol{v}_{1}(t, \boldsymbol{x}), \ldots, \boldsymbol{v}_{N}(t, \boldsymbol{x}), \gamma_{t}=m\right) \\
& =\sum_{i=1}^{N}\left\|\boldsymbol{v}_{i}(t, \boldsymbol{x})\right\|^{2}+\sum_{i=1}^{N} \frac{1}{\rho_{i}}\left(k_{i}(t)-\tilde{k}\right)^{2}
\end{aligned}
$$

where $\tilde{k}$ is a positive constant to be determined. We denote $V\left(t, \boldsymbol{v}_{1}(t, \boldsymbol{x}), \ldots, \boldsymbol{v}_{N}(t, \boldsymbol{x}), \gamma_{t}=m\right)$ by $V(t)$. Thanks to Itô formula, we have

$$
\begin{aligned}
\mathrm{d} V(t)= & \sum_{i=1}^{N} \mathcal{L} V_{i} \mathrm{~d} t+2 \sum_{i=1}^{N}\left(k_{i}(t)-\tilde{k}\right)\left\|\boldsymbol{v}_{i}(t, \boldsymbol{x})\right\|^{2} \mathrm{~d} t \\
& +2 \sum_{i=1}^{N} \int_{\mathbb{O}} \boldsymbol{v}_{i}^{T} \boldsymbol{\sigma} \mathrm{d} \boldsymbol{w} \mathrm{d} \boldsymbol{x}
\end{aligned}
$$

where

$$
\begin{aligned}
\mathcal{L} V_{i}= & \int_{\mathbb{Q}} \boldsymbol{v}_{i}^{T}(t, \boldsymbol{x})\left(\nabla \cdot\left(\boldsymbol{D}(\boldsymbol{x}) \circ \nabla \boldsymbol{v}_{i}(t, \boldsymbol{x})\right)\right) \mathrm{d} \boldsymbol{x} \\
& +\int_{\mathbb{O}}\left(\nabla \cdot\left(\boldsymbol{D}(\boldsymbol{x}) \circ \nabla \boldsymbol{v}_{i}(t, \boldsymbol{x})\right)\right)^{T} \boldsymbol{v}_{i}(t, \boldsymbol{x}) \mathrm{d} \boldsymbol{x} \\
& -\int_{\mathbb{O}}\left[\boldsymbol{v}_{i}^{T}(t, \boldsymbol{x}) \boldsymbol{A}_{m} \boldsymbol{v}_{i}(t, \boldsymbol{x})+\boldsymbol{v}_{i}(t, \boldsymbol{x})^{T} \boldsymbol{A}_{m}^{T} \boldsymbol{v}_{i}(t, \boldsymbol{x})\right] \mathrm{d} \boldsymbol{x} \\
& +\int_{\mathbb{O}}\left[\boldsymbol{v}_{i}^{T}(t, \boldsymbol{x}) \boldsymbol{B}_{1 m} \boldsymbol{F}\left(\boldsymbol{v}_{i}\right)+\boldsymbol{F}\left(\boldsymbol{v}_{i}\right)^{T} \boldsymbol{B}_{1 m}^{T} \boldsymbol{v}_{i}(t, \boldsymbol{x})\right] \mathrm{d} \boldsymbol{x} \\
& +\int_{\mathbb{O}}\left[\boldsymbol{v}_{i}^{T}(t, \boldsymbol{x}) \boldsymbol{B}_{2 m} \boldsymbol{F}\left(\mathcal{S}\left(\boldsymbol{v}_{i}\right)\right)+\boldsymbol{F}\left(\mathcal{S}\left(\boldsymbol{v}_{i}\right)\right)^{T} \boldsymbol{B}_{2 m}^{T} \boldsymbol{v}_{i}(t, \boldsymbol{x})\right] \mathrm{d} \boldsymbol{x} \\
& +\int_{\mathbb{O}} \boldsymbol{v}_{i}^{T}(t, \boldsymbol{x})\left[\sum_{j=1}^{N} c_{i j} \boldsymbol{G}_{m}\left(\boldsymbol{v}_{j}+\mathcal{S}\left(\boldsymbol{v}_{j}\right)\right)\right] \mathrm{d} \boldsymbol{x} \\
& +\int_{\mathbb{O}}\left[\sum_{j=1}^{N} c_{i j} \boldsymbol{G}_{m}\left(\boldsymbol{v}_{j}+\mathcal{S}\left(\boldsymbol{v}_{j}\right)\right)\right]^{T} \boldsymbol{v}_{i}(t, \boldsymbol{x}) \mathrm{d} \boldsymbol{x} \\
& +\int_{\mathbb{O}} \boldsymbol{v}_{i}^{T}(t, \boldsymbol{x}) \boldsymbol{U}_{i}(t, \boldsymbol{x}) \mathrm{d} \boldsymbol{x}-\int_{\mathbb{O}} \boldsymbol{U}_{i}^{T}(t, \boldsymbol{x}) \boldsymbol{v}_{i}(t, \boldsymbol{x}) \mathrm{d} \boldsymbol{x} \\
& \left.\int_{\mathbb{O}} \boldsymbol{\sigma}{ }^{T} \boldsymbol{\sigma} \mathrm{d} \boldsymbol{x}\right) \triangleq \sum_{k=1} \mathcal{L} V_{i k} \cdot
\end{aligned}
$$

Taking expectation of $\mathrm{d} V(t)$, we know that

$$
\frac{\mathrm{d} \mathcal{E} V(t)}{\mathrm{d} t}=\sum_{i=1}^{N} \mathcal{E} \mathcal{L} V_{i}+2 \sum_{i=1}^{N}\left(k_{i}(t)-\tilde{k}\right) \mathcal{E}\left\|\boldsymbol{v}_{i}(t, \boldsymbol{x})\right\|^{2} \text {. }
$$

From Gauss formula, Dirichlet boundary condition and Poincaré inequality, we have 


$$
\begin{aligned}
& \sum_{k=1}^{l} \int_{\Omega} v_{i j} \frac{\partial}{\partial x_{k}}\left(D_{i k} \frac{\partial v_{i j}}{\partial x_{k}}\right) \mathrm{d} \boldsymbol{x} \\
& \quad \leq \int_{\partial \Omega}\left(v_{i j} D_{i k} \frac{\partial v_{i j}}{\partial x_{k}}\right)_{k=1}^{l} \cdot \mathbf{n} \mathrm{d} \boldsymbol{x}-\int_{\Omega} \sum_{k=1}^{l} D_{i k}\left(\frac{\partial v_{i j}}{\partial x_{k}}\right)^{2} \mathrm{~d} \boldsymbol{x} \\
& \quad \leq-\sum_{k=1}^{l} \int_{\Omega} \hat{D}_{i k}\left(\frac{\partial v_{i j}}{\partial x_{k}}\right)^{2} \mathrm{~d} \boldsymbol{x},
\end{aligned}
$$

where $i=1,2, \ldots, N$ and $j=1,2, \ldots, n$, furthermore,

$\mathcal{E} \mathcal{L} V_{i 1}+\mathcal{E} \mathcal{L} V_{i 2} \leq-\alpha^{2} \mathcal{E} \int_{\Omega} \boldsymbol{v}_{i}^{T}(t, \boldsymbol{x})\left(\hat{\boldsymbol{D}}+\hat{\boldsymbol{D}}^{T}\right) \boldsymbol{v}_{i}(t, \boldsymbol{x}) \mathrm{d} \boldsymbol{x}$.

From Lemma 2, we obtain

$$
\begin{aligned}
\mathcal{E} \mathcal{L} V_{i 4} \leq & \mathcal{E} \int_{\Omega} \boldsymbol{v}_{i}^{T}(t, \boldsymbol{x}) \boldsymbol{B}_{1 m} \boldsymbol{B}_{1 m}^{T} \boldsymbol{v}_{i}(t, \boldsymbol{x}) \mathrm{d} \boldsymbol{x} \\
& +L^{2} \mathcal{E} \int_{\Omega} \boldsymbol{v}_{i}^{T}(t, \boldsymbol{x}) \boldsymbol{v}_{i}(t, \boldsymbol{x}) \mathrm{d} \boldsymbol{x},
\end{aligned}
$$

and

$$
\begin{aligned}
\mathcal{E} \mathcal{L} V_{i 5} & \leq \mathcal{E} \int_{\Omega} \boldsymbol{v}_{i}^{T}(t, \boldsymbol{x}) \boldsymbol{B}_{2 m} \boldsymbol{B}_{2 m}^{T} \boldsymbol{v}_{i}(t, \boldsymbol{x}) \mathrm{d} \boldsymbol{x}+\mathcal{E} \int_{\Omega} \boldsymbol{F}^{T}\left(\mathcal{S}\left(\boldsymbol{v}_{i}\right)\right) \boldsymbol{F}\left(\mathcal{S}\left(\boldsymbol{v}_{i}\right)\right) \mathrm{d} \boldsymbol{x} \\
& \leq \mathcal{E} \int_{\Omega} \boldsymbol{v}_{i}^{T}(t, \boldsymbol{x}) \boldsymbol{B}_{2 m} \boldsymbol{B}_{2 m}^{T} \boldsymbol{v}_{i}(t, \boldsymbol{x}) \mathrm{d} \boldsymbol{x}+L^{2} \mathcal{E}\left\|\int_{-\infty}^{0} \mathrm{~d} \boldsymbol{\eta}(\theta) \boldsymbol{v}_{i}(t+\theta, \boldsymbol{x})\right\|^{2} .
\end{aligned}
$$

Since $\int_{-\infty}^{0} d \eta_{i j}(\theta)=\hat{\eta}_{i j}>0(i, j=1,2, \ldots, n)$ and $\eta_{i j}(\theta)$ is non-decreasing bounded variation function, there exists a constant $\tau \geq t$ such that $\int_{-\infty}^{-\tau} d \eta_{i j}(\theta) \leq \epsilon$ for any $\epsilon \geq 0$ and $i, j=1,2, \ldots, n$. So,

$$
\begin{aligned}
& \mathcal{E}\left\|\int_{-\infty}^{0} \mathrm{~d} \boldsymbol{\eta}(\theta) \boldsymbol{v}_{i}(t+\theta, \boldsymbol{x})\right\|^{2} \\
& \leq 2 \mathcal{E}\left\|\int_{-\tau}^{0} \mathrm{~d} \boldsymbol{\eta}(\theta) \boldsymbol{v}_{i}(t+\theta, \boldsymbol{x})\right\|^{2}+2 \mathcal{E}\left\|\int_{-\infty}^{-\tau} \mathrm{d} \boldsymbol{\eta}(\theta) \boldsymbol{v}_{i}(t+\theta, \boldsymbol{x})\right\|^{2} \\
& \leq 2\|\hat{\boldsymbol{\eta}}\|_{F}^{2} \sup _{-\tau \leq \theta \leq 0} \mathcal{E}\left\|\boldsymbol{v}_{i}(t+\theta, \boldsymbol{x})\right\|^{2}+2 \mathcal{E}\left\|\boldsymbol{\phi}_{i}\right\|_{\mathbb{C}}^{2} \epsilon^{2},
\end{aligned}
$$

and

$$
\begin{aligned}
\mathcal{E} \mathcal{L} V_{i 5} \leq & \mathcal{E} \int_{\Omega} \boldsymbol{v}_{i}^{T}(t, \boldsymbol{x}) \boldsymbol{B}_{2 m} \boldsymbol{B}_{2 m}^{T} \boldsymbol{v}_{i}(t, \boldsymbol{x}) \mathrm{d} \boldsymbol{x} \\
& +2\|\hat{\boldsymbol{\eta}}\|_{F}^{2} L^{2} \sup _{-\tau \leq \theta \leq 0} \mathcal{E}\left\|\boldsymbol{v}_{i}(t+\theta, \boldsymbol{x})\right\|^{2}+2 L^{2} \mathcal{E}\left\|\boldsymbol{\phi}_{i}\right\|_{\mathbb{C}}^{2} \epsilon^{2} .
\end{aligned}
$$

Obviously,

$$
\begin{aligned}
\mathcal{E} \mathcal{L} V_{i 6} & =\sum_{j=1}^{N} \mathcal{E} \int_{\mathbb{Q}} \boldsymbol{v}_{i}^{T}(t, \boldsymbol{x}) c_{i j} \boldsymbol{G}_{m} \boldsymbol{v}_{j}(t, \boldsymbol{x}) \mathrm{d} \boldsymbol{x} \\
& +\sum_{j=1}^{N} \mathcal{E} \int_{\mathbb{O}} \boldsymbol{v}_{i}^{T}(t, \boldsymbol{x}) c_{i j} \boldsymbol{G}_{m} \mathcal{S}\left(\boldsymbol{v}_{j}\right) \mathrm{d} \boldsymbol{x}, \\
\mathcal{E} \mathcal{L} V_{i\urcorner} & =\sum_{j=1}^{N} \mathcal{E} \int_{\mathbb{O}} \boldsymbol{v}_{j}^{T}(t, \boldsymbol{x}) c_{i j} \boldsymbol{G}_{m}^{T} \boldsymbol{v}_{i}(t, \boldsymbol{x}) \mathrm{d} \boldsymbol{x} \\
& +\sum_{j=1}^{N} \mathcal{E} \int_{\mathbb{O}} \mathcal{S}^{T}\left(\boldsymbol{v}_{j}\right) c_{i j} \boldsymbol{G}_{m}^{T} \boldsymbol{v}_{i}(t, \boldsymbol{x}) \mathrm{d} \boldsymbol{x} .
\end{aligned}
$$

From Lemma 2, we get

$$
\begin{aligned}
& \int_{\mathbb{O}}\left[\boldsymbol{v}_{i}^{T}(t, \boldsymbol{x}) c_{i j} \boldsymbol{G}_{m} \boldsymbol{v}_{j}(t, \boldsymbol{x})+\boldsymbol{v}_{j}^{T}(t, \boldsymbol{x}) c_{i j} \boldsymbol{G}_{m}^{T} \boldsymbol{v}_{i}(t, \boldsymbol{x})\right] \mathrm{d} \boldsymbol{x} \\
& \quad \leq \int_{\mathbb{O}}\left[\boldsymbol{v}_{i}^{T}(t, \boldsymbol{x}) \boldsymbol{G}_{m} \boldsymbol{G}_{m}^{T} \boldsymbol{v}_{i}(t, \boldsymbol{x})+c_{i j}^{2} \boldsymbol{v}_{j}^{T}(t, \boldsymbol{x}) \boldsymbol{v}_{j}(t, \boldsymbol{x})\right] \mathrm{d} \boldsymbol{x},
\end{aligned}
$$

and

$$
\begin{aligned}
& \int_{\mathbb{Q}} \boldsymbol{v}_{i}^{T}(t, \boldsymbol{x}) c_{i j} \boldsymbol{G}_{m} \mathcal{S}\left(\boldsymbol{v}_{j}\right) \mathrm{d} \boldsymbol{x}+\int_{\mathbb{Q}} \mathcal{S}^{T}\left(\boldsymbol{v}_{j}\right) c_{i j} \boldsymbol{G}_{m}^{T} \boldsymbol{v}_{i}(t, \boldsymbol{x}) \mathrm{d} \boldsymbol{x} \\
& \leq \int_{\mathbb{Q}} \boldsymbol{v}_{i}^{T}(t, \boldsymbol{x}) \boldsymbol{G}_{m} \boldsymbol{G}_{m}^{T} \boldsymbol{v}_{i}(t, \boldsymbol{x}) \mathrm{d} \boldsymbol{x}+c_{i j}^{2}\left\|\int_{-\infty}^{0} \mathrm{~d} \boldsymbol{\eta}(\theta) \boldsymbol{v}_{j}(t+\theta, \boldsymbol{x})\right\|^{2} .
\end{aligned}
$$

Thus,

$$
\begin{gathered}
\sum_{i=1}^{N}\left(\mathcal{E} \mathcal{L} V_{i 6}+\mathcal{E} \mathcal{L} V_{i 7}\right) \leq \sum_{i=1}^{N} \mathcal{E} \int_{\Omega} \boldsymbol{v}_{i}^{T}(t, \boldsymbol{x})\left(2 N \boldsymbol{G}_{m} \boldsymbol{G}_{m}^{T}\right. \\
\left.+\sum_{j=1}^{N} c_{j}^{2}\right) \boldsymbol{v}_{i}(t, \boldsymbol{x}) \mathrm{d} \boldsymbol{x}+2\|\hat{\boldsymbol{\eta}}\|_{F}^{2} \sum_{j=1}^{N} c_{j}^{2} \sup _{-\tau \leq \theta \leq 0} \\
\mathcal{E} \sum_{i=1}^{N}\left\|\boldsymbol{v}_{i}(t+\theta, \boldsymbol{x})\right\|^{2}+2 \sum_{j=1}^{N} c_{j}^{2} \sum_{i=1}^{N} \mathcal{E}\left\|\boldsymbol{\phi}_{i}\right\|_{\mathbb{C}}^{2} \epsilon^{2},
\end{gathered}
$$

where $c_{j}=\max _{i=1,2, \ldots, n}\left\{c_{j i}\right\}$. Besides,

$$
\begin{aligned}
& \mathcal{E} \mathcal{L} V_{i 9} \leq L^{2}\left(\mathcal{E}\left\|\boldsymbol{v}_{i}(t, \boldsymbol{x})\right\|^{2}+2\|\hat{\boldsymbol{\eta}}\|_{F}^{2} \sup _{-\tau \leq \theta \leq 0} \mathcal{E}\left\|\boldsymbol{v}_{i}(t+\theta, \boldsymbol{x})\right\|^{2}\right. \\
& \left.+2 \mathcal{E}\left\|\boldsymbol{\phi}_{i}\right\|_{\mathbb{C}}^{2} \epsilon^{2}\right) .
\end{aligned}
$$

Thus, combining (11)-(23), we have

$$
\frac{\mathrm{d} \mathcal{E} V(t)}{\mathrm{d} t} \leq\left(\beta_{m}-\tilde{k}\right) \mathcal{E} V(t)+b \sup _{-\tau \leq \theta \leq 0} \mathcal{E} V(t+\theta)+c \epsilon^{2},
$$

where $\beta_{m}$ is the maximum eigenvalue of $\boldsymbol{\Lambda}_{m}, \boldsymbol{\Lambda}_{m}=-\alpha^{2}$ $\hat{\boldsymbol{D}}-\alpha^{2} \hat{\boldsymbol{D}}^{T}-\boldsymbol{A}_{m}-\boldsymbol{A}_{m}^{T}+\boldsymbol{B}_{1 m} \boldsymbol{B}_{1 m}^{T}+\boldsymbol{B}_{2 m} \boldsymbol{B}_{2 m}^{T}+2 N \boldsymbol{G}_{m} \boldsymbol{G}_{m}^{T}+$ 
$\left(2 L^{2}+\sum_{i=1}^{N} c_{i}^{2}\right) \boldsymbol{I}, \quad b=2\left(2 L^{2}+\sum_{i=1}^{N} c_{i}^{2}\right)\|\hat{\boldsymbol{\eta}}\|_{F}^{2} \quad$ and $\quad c=$ $2\left(2 L^{2}+\sum_{j=1}^{N} c_{j}^{2}\right) \mathcal{E} \sum_{i=1}^{N}\left\|\boldsymbol{\phi}_{i}\right\|_{\mathbb{C}}^{2}$. The constant $\tilde{k}$ can be properly chosen as $\tilde{k}=\max _{m}\left\{0, \beta_{m}+b+1\right\}$, then one can get $\quad \beta_{m}-\tilde{k}+b<0 . \quad$ Besides, $\quad \mathcal{E} \sum_{i=1}^{N}\left\|\boldsymbol{v}_{i}(t, \boldsymbol{x})\right\|^{2}$ $\leq \mathcal{E} \sum_{i=1}^{N}\left\|\boldsymbol{\phi}_{i}\right\|_{\mathbb{C}}^{2}$ for $t \in(-\infty, 0]$. From Lemma 5, we see that there exists a positive constant $\lambda$ such that $\mathcal{E} \sum_{i=1}^{N}\left\|\boldsymbol{v}_{i}(t, \boldsymbol{x})\right\|^{2} \leq e^{-\lambda t} \mathcal{E} \sum_{i=1}^{N}\left\|\boldsymbol{\phi}_{i}\right\|_{\mathbb{C}}^{2}$. Then, the drive system (2) and response system (3) are globally exponentially synchronous in mean square.

Remark 2 When $\boldsymbol{\sigma}\left(\varsigma_{1}, \varsigma_{2}\right)=0$, the error system (4) becomes Markovian jumping complex dynamical networks with reaction-diffusion terms and S-type distributed delays which include Markovian jumping networks and deterministic networks with time-varying delays [39, 48].

As we know, the S-type distributed delays include timevarying delays and distributed time delays. Then, we have Corollary 1 for SCDNs (25) with reaction-diffusion terms and mixed time delays

$$
\begin{aligned}
\mathrm{d} \boldsymbol{v}_{i}=[\nabla & \left.\cdot\left(\tilde{\boldsymbol{D}}(\boldsymbol{x}) \circ \nabla \boldsymbol{v}_{i}\right)-\tilde{\boldsymbol{A}}_{m} \boldsymbol{v}_{i}+\tilde{\boldsymbol{F}}\left(\boldsymbol{v}_{i}\right)+\tilde{\boldsymbol{J}}\left(\boldsymbol{v}_{i}\right)+\boldsymbol{U}_{i}(t, \boldsymbol{x})\right] \mathrm{d} t \\
& +\tilde{\boldsymbol{\sigma}}\left(\boldsymbol{v}_{i}, \mathcal{M}_{1}\left(\boldsymbol{v}_{i}\right), \mathcal{M}_{2}\left(\boldsymbol{v}_{i}\right)\right) \mathrm{d} \boldsymbol{w}(t),
\end{aligned}
$$

where

$$
\begin{aligned}
& \tilde{\boldsymbol{F}}\left(\boldsymbol{v}_{i}\right)=\tilde{\boldsymbol{B}}_{1 m} \boldsymbol{F}\left(\boldsymbol{v}_{i}(t, \boldsymbol{x})\right)+\tilde{\boldsymbol{B}}_{2 m} \boldsymbol{F}\left(\mathcal{M}_{1}\left(\boldsymbol{v}_{i}\right)\right)+\tilde{\boldsymbol{B}}_{3 m} \boldsymbol{F}\left(\mathcal{M}_{2}\left(\boldsymbol{v}_{i}\right)\right) \\
& \tilde{\boldsymbol{J}}\left(\boldsymbol{v}_{i}\right)=\sum_{j=1}^{N} c_{i j} \tilde{\boldsymbol{G}}_{m}\left(\boldsymbol{v}_{j}+\mathcal{M}_{1}\left(\boldsymbol{v}_{j}\right)+\mathcal{M}_{2}\left(\boldsymbol{v}_{j}\right)\right) \\
& \mathcal{M}_{1}\left(\boldsymbol{v}_{i}\right)=\boldsymbol{v}_{i}(t-\tau(t), \boldsymbol{x}) \\
& \mathcal{M}_{2}\left(\boldsymbol{v}_{i}\right)=\int_{-\infty}^{0} \zeta(-\theta) \boldsymbol{v}_{i}(t+\theta, \boldsymbol{x}) \mathrm{d} \theta
\end{aligned}
$$

Corollary 1 If the following conditions hold,

(i) $0 \leq \tau(t) \leq \tilde{\tau}, 0 \leq \zeta(t) \leq \kappa e^{-\lambda t}$ where $t \geq 0, \tilde{\tau}, \kappa$, and $\lambda$ are positive constants;

(ii) $\quad\left\|\tilde{\boldsymbol{\sigma}}\left(\varsigma_{1}, \varsigma_{2}, \varsigma_{3}\right)\right\|^{2} \leq L^{2}\left(\left\|\varsigma_{1}\right\|^{2}+\left\|\varsigma_{2}\right\|^{2}+\left\|\varsigma_{3}\right\|^{2}\right)$, $\varsigma_{1}, \varsigma_{2}, \varsigma_{3} \in \mathbb{R}^{n}$,

then the error system (25) and the corresponding driveresponse system with adaptive controller (7) are exponentially synchronous in mean square.

From Theorem 1, SCDNs can synchronize by adaptive control strategy on the whole networks. However, it is sometimes impractical to impose control inputs on all nodes of SCDNs, especially large-scale SCDNs. To reduce the number of controlled nodes, pinning control is applied to a fraction of networks. The adaptive pinning controller can be expressed by
$\boldsymbol{U}_{i}(t, \boldsymbol{x})= \begin{cases}-k_{i}(t) \boldsymbol{v}_{i}(t, \boldsymbol{x}), & i=1,2, \ldots, p \\ \boldsymbol{0}, & i=p+1, \ldots, N,\end{cases}$

where the first $p$ nodes are pinned with adaptive controller and the adaptive law is designed as $\dot{k}_{i}(t)=\rho_{i}\left\|\boldsymbol{v}_{i}(t, \boldsymbol{x})\right\|^{2}$ $(i=1,2, \ldots, N)$. Then, we have the following result.

Theorem 2 The drive system (2) and response system (3) with adaptive pinning controller (26) are exponentially synchronous in mean square, if $\bar{\beta}+\lambda_{p+1}+b<0$ where $\bar{\beta}$ is the maximum eigenvalue of $\overline{\boldsymbol{\Lambda}}_{m}, \overline{\boldsymbol{\Lambda}}_{m}=-\alpha^{2} \hat{\boldsymbol{D}}-\alpha^{2} \hat{\boldsymbol{D}}^{T}-$ $\boldsymbol{A}_{m}-\boldsymbol{A}_{m}^{T}+\boldsymbol{B}_{1 m} \boldsymbol{B}_{1 m}^{T}+\boldsymbol{B}_{2 m} \boldsymbol{B}_{2 m}^{T}+2 N \boldsymbol{G}_{m} \boldsymbol{G}_{m}^{T}+2 L^{2} \boldsymbol{I}_{n} \quad$ for $m \in \mathbb{M}, \quad \lambda_{1} \geq \lambda_{2} \geq \cdots \geq \lambda_{N}$ are the eigenvalues of the matrix $\quad \overline{\boldsymbol{C}}, \quad \overline{\boldsymbol{C}}=\sum_{j=1}^{N} \operatorname{diag}\left(c_{j 1}, c_{j 2} \cdots, c_{j N}\right), \quad$ and $b=2\left(2 L^{2}+\sum_{i=1}^{N} c_{i}^{2}\right)\|\hat{\boldsymbol{\eta}}\|_{F}^{2}$.

Proof Consider the following Lyapunov-Krasovskii functional

$\bar{V}(t)=\sum_{i=1}^{N}\left\|\boldsymbol{v}_{i}(t, \boldsymbol{x})\right\|^{2}+\sum_{i=1}^{p} \frac{1}{\rho_{i}}\left(k_{i}(t)-\bar{k}\right)^{2}$,

where $\bar{k}=\lambda_{1}-\lambda_{p+1}$. As (8)-(24), we know that

$\frac{\mathrm{d} \mathcal{E} \bar{V}(t)}{\mathrm{d} t} \leq \mathcal{E} \int_{\Omega} \overline{\boldsymbol{v}}^{T}(t, \boldsymbol{x}) \boldsymbol{\Xi} \overline{\boldsymbol{v}}(t, \boldsymbol{x}) \mathrm{d} \boldsymbol{x}+b \sup _{-\tau \leq \theta \leq 0} \mathcal{E} \bar{V}(t+\theta)+c \epsilon^{2}$,

where

$$
\begin{aligned}
& \boldsymbol{\Xi}=\boldsymbol{I}_{N} \otimes \overline{\boldsymbol{\Lambda}}_{m}+\overline{\boldsymbol{C}} \otimes \boldsymbol{I}_{n}-2 \overline{\boldsymbol{K}} \otimes \boldsymbol{I}_{n}, \\
& \overline{\boldsymbol{v}}(t, \boldsymbol{x})=\left[\boldsymbol{v}_{1}^{T}(t, \boldsymbol{x}), \boldsymbol{v}_{2}^{T}(t, \boldsymbol{x}), \ldots, \boldsymbol{v}_{N}^{T}(t, \boldsymbol{x})\right]^{T}, \\
& \boldsymbol{I}_{N}=\operatorname{diag}(\underbrace{1, \ldots, 1}_{N}), \boldsymbol{I}_{n}=\operatorname{diag}(\underbrace{1, \ldots, 1}_{n}), \\
& \overline{\boldsymbol{K}}=\operatorname{diag}(\underbrace{\bar{k}, \ldots, \bar{k}}_{p}, \underbrace{0, \ldots, 0}_{N-p}),
\end{aligned}
$$

and $\otimes$ denotes the Kronecker product. Since $\overline{\boldsymbol{C}}-2 \overline{\boldsymbol{K}}$ is a symmetric matrix, there exists an orthogonal matrix $\boldsymbol{O}$ satisfying $\quad \overline{\boldsymbol{C}}-2 \overline{\boldsymbol{K}}=\boldsymbol{O}^{T} \operatorname{diag}\left(\bar{\lambda}_{1}, \ldots, \bar{\lambda}_{N}\right) \boldsymbol{O} \quad$ where $\bar{\lambda}_{1} \geq \bar{\lambda}_{2} \geq \cdots, \bar{\lambda}_{N}$ are the eigenvalues of the matrix $\overline{\boldsymbol{C}}-2 \overline{\boldsymbol{K}}$. According to Lemma 3, one has

$$
\begin{cases}-2 \bar{k}+\lambda_{N} \leq \bar{\lambda}_{i} \leq-2 \bar{k}+\lambda_{1} & 1 \leq i \leq p ; \\ -2 \bar{k}+\lambda_{i} \leq \bar{\lambda}_{i} \leq \lambda_{i} & p+1 \leq i \leq N .\end{cases}
$$

Thus,

$$
\begin{aligned}
\frac{\mathrm{d} \mathcal{E} \bar{V}(t)}{\mathrm{d} t} & \leq \mathcal{E} \int_{\Omega} \overline{\boldsymbol{v}}^{T}(t, \boldsymbol{x}) \boldsymbol{O}^{T} \overline{\boldsymbol{H}}_{1} \boldsymbol{O} \otimes \boldsymbol{I}_{n} \overline{\boldsymbol{v}}(t, \boldsymbol{x}) \mathrm{d} \boldsymbol{x}+b \sup _{-\tau \leq \theta \leq 0} \mathcal{E} \bar{V}(t+\theta)+c \epsilon^{2}, \\
& \leq \mathcal{E} \int_{\Omega} \overline{\boldsymbol{v}}^{T}(t, \boldsymbol{x}) \boldsymbol{O}^{T} \overline{\boldsymbol{H}}_{2} \boldsymbol{O} \otimes \boldsymbol{I}_{n} \overline{\boldsymbol{v}}(t, \boldsymbol{x}) \mathrm{d} \boldsymbol{x}+b \sup _{-\tau \leq \theta \leq 0} \mathcal{E} \bar{V}(t+\theta)+c \epsilon^{2} \\
& \leq\left(\bar{\beta}+\lambda_{p+1}\right) \mathcal{E} \bar{V}(t)+b \sup _{-\tau \leq \theta \leq 0} \mathcal{E} \bar{V}(t+\theta)+c \epsilon^{2},
\end{aligned}
$$


where

$$
\begin{aligned}
& \overline{\boldsymbol{H}}_{1}=\operatorname{diag}\left(\bar{\beta}+\bar{\lambda}_{1}, \ldots, \bar{\beta}+\bar{\lambda}_{N}\right), \\
& \overline{\boldsymbol{H}}_{2}=\operatorname{diag}(\underbrace{\bar{\beta}-2 \bar{k}+\lambda_{1}, \ldots, \bar{\beta}-2 \bar{k}+\lambda_{1}}_{p}, \underbrace{\left.\bar{\beta}+\lambda_{p+1}, \cdots, \bar{\beta}+\lambda_{N}\right)}_{N-p}, \\
& c=2\left(2 L^{2}+\sum_{j=1}^{N} c_{j}^{2}\right) \mathcal{E} \sum_{i=1}^{N}\left\|\boldsymbol{\phi}_{i}\right\|_{\mathbb{C}}^{2} .
\end{aligned}
$$

From Lemma 5 and $\bar{\beta}+\lambda_{p+1}+b<0$, we know that the drive system (2) and response system (3) are globally exponentially synchronous in mean square.

Remark 3 Most of the existing criteria are established for systems with finite delays [1, 14, 26], whereas adaptive control and adaptive pinning control strategies in this paper are derived for stochastic systems with reactiondiffusion terms and S-type distributed delays which are infinite delays. Due to the existence of reaction-diffusion terms and S-type distributed delays, inequality with infinite delay is derived for the synchronization criteria in Hilbert space.

Remark 4 In most of existing works about distributed time delays [2, 4, 18, 19, 48], a Lyapunov-Krasovskii functional candidate with distributed term is usually constructed. In contrast, as the infinite delays here are handled by a generalized Halanay inequality and truncation method, the criteria in this paper are obtained by constructing a simple Lyapunov-Krasovskii functional candidate without the distributed terms.

\section{Numerical examples}

In this section, some numerical examples are given to illustrate the effectiveness of the obtained results.

Example 1 Consider a 2-D SCDN with mixed time delays whose parameters are taken as follows: $\tilde{\boldsymbol{D}}=0$, $\mathbb{M}=\{1,2\}$,

$$
\begin{aligned}
\tilde{\boldsymbol{A}}_{1}= & {\left[\begin{array}{cc}
1.1 & 0 \\
0 & 1.2
\end{array}\right], \tilde{\boldsymbol{A}}_{2}=\left[\begin{array}{cc}
1.2 & 0 \\
0 & 1.5
\end{array}\right], \tilde{\boldsymbol{B}}_{11}=\left[\begin{array}{cc}
1.2 & -1.5 \\
-1.7 & 1.2
\end{array}\right], } \\
\tilde{\boldsymbol{B}}_{12}= & {\left[\begin{array}{cc}
0.6 & -0.1 \\
-0.1 & 0.2
\end{array}\right], \tilde{\boldsymbol{B}}_{21}=\left[\begin{array}{cc}
0.5 & 0 \\
0 & 0.5
\end{array}\right], \tilde{\boldsymbol{B}}_{22}=\left[\begin{array}{cc}
0.8 & 0 \\
0 & 0.8
\end{array}\right], } \\
\tilde{\boldsymbol{B}}_{31}= & {\left[\begin{array}{cc}
1.1 & 0.5 \\
0.5 & 0.8
\end{array}\right], \tilde{\boldsymbol{B}}_{32}=\left[\begin{array}{cc}
0.8 & 0.2 \\
0.2 & 0.3
\end{array}\right], \tilde{\boldsymbol{G}}_{1}=\left[\begin{array}{cc}
1 & -0.5 \\
0.5 & 1
\end{array}\right], } \\
& \tilde{\boldsymbol{G}}_{2}=\left[\begin{array}{cc}
0.1 & 0.05 \\
0.05 & 0.1
\end{array}\right] .
\end{aligned}
$$

The delay and activation functions are taken as

$$
\begin{aligned}
& \boldsymbol{f}\left(\boldsymbol{\varsigma}_{1}\right)=\frac{1}{2}\left(\left|\varsigma_{1}+1\right|+\left|\varsigma_{1}-1\right|\right), \tilde{\boldsymbol{g}}\left(\boldsymbol{\varsigma}_{1}, \varsigma_{2}, \varsigma_{3}\right)=\tanh \left(\varsigma_{3}\right), \\
& \boldsymbol{I}(t)=\left[e^{-t} \cos (t), \sin (t)\right]^{T} \\
& \mathcal{M}_{1}(\boldsymbol{s})=\boldsymbol{s}\left(t-\frac{1}{2}|\sin (t)|, \boldsymbol{x}\right) \\
& \mathcal{M}_{2}(\boldsymbol{s})=\int_{-\infty}^{0} e^{\theta} \boldsymbol{s}(t+\theta, \boldsymbol{x}) \mathrm{d} \theta
\end{aligned}
$$

The response system consists of 10 coupled identical nodes with structure shown in Fig. 1a. The initial conditions are chosen as $s(\theta)=[1.5,0.5]^{T}$ for drive system and proper negative constants for 10 nodes of the response system for $\theta \in[-1,0]$. From Corollary 1, the drive-response systems with adaptive controller (7) are exponentially synchronous. Figure 2 depicts the time trajectory of drive-response system and the error system (node 6) without control. From Fig. 2, we see that the drive signal (red line in Fig. 2a) and the response signal (blue line in Fig. 2a) have different dynamical behaviors and the error signal (green line in Fig. 2b) does not evolve toward zero. Thus, the uncontrolled system is not synchronous. In contrast, Fig. 3 illustrates the controlled systems with adaptive controller. Obviously, the drive and response signals in Fig. 3a share the same patterns and the error signal in Fig. $3 \mathrm{~b}$ becomes zero after 8 . Therefore, the system with the adaptive controller is synchronous, which corresponds to the theoretical analysis.

Example 2 Then, we consider a 1-D SCDN with reactiondiffusion terms and time delays. The parameters and functions are taken as $D=0.1, \mathbb{M}=\{1,2\}, A_{1}=0.05$, $A_{2}=0.1, B_{11}=0.4, B_{12}=0.35, B_{21}=0.3, B_{22}=0.4$, $G_{1}=1, G_{2}=0.1, f\left(\varsigma_{1}\right)=\tanh \left(\varsigma_{1}\right), g\left(\varsigma_{1}, \varsigma_{2}\right)=f\left(\varsigma_{1}+\varsigma_{2}\right)$, $I(t)=\sin (t), \quad \mathcal{S}(s)=s(t-\tau(t), x)$ and $\tau(t)=\frac{e^{t}}{e^{t}+1}$. The response system consists of 5 identical nodes with structure shown in Fig. 1b. From Theorem 1, we know that the drive-response system with adaptive controller (7) can be exponentially synchronous. The dynamical behaviors of uncontrolled error system and controlled one (node 5) are shown in Fig. 4.

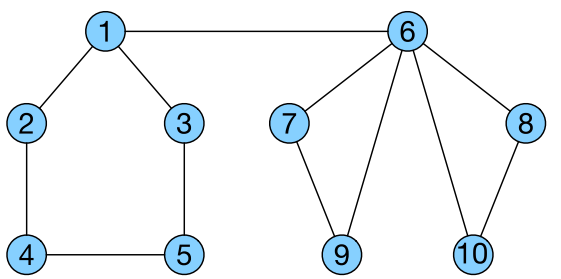

(a)

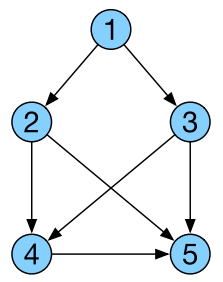

(b)
Fig. 1 The network topologies in Example 1 (left) and 2 (right) 


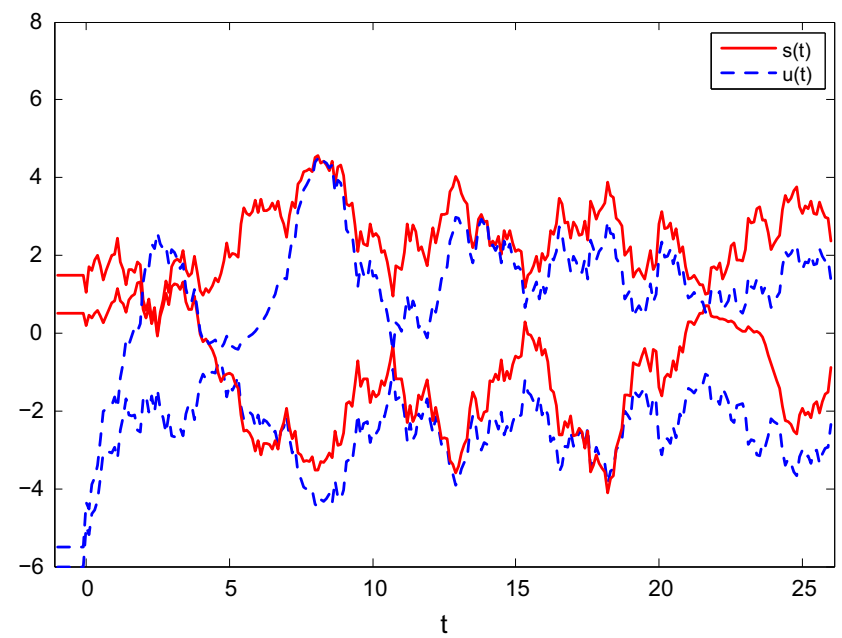

(a)

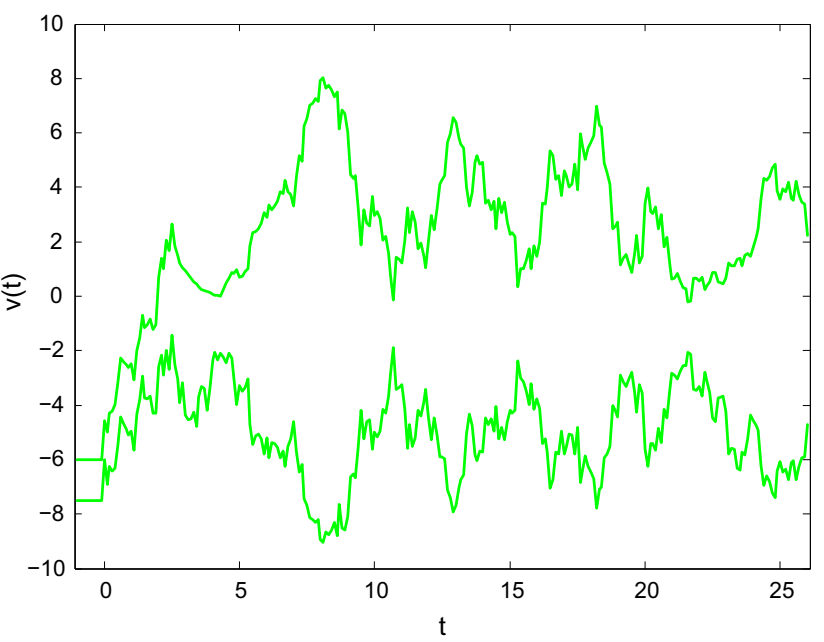

(b)

Fig. 2 Time trajectory of the uncontrolled system (node 6) in Example 1. a The drive signal (red line) and response signal (blue line). b The error signal

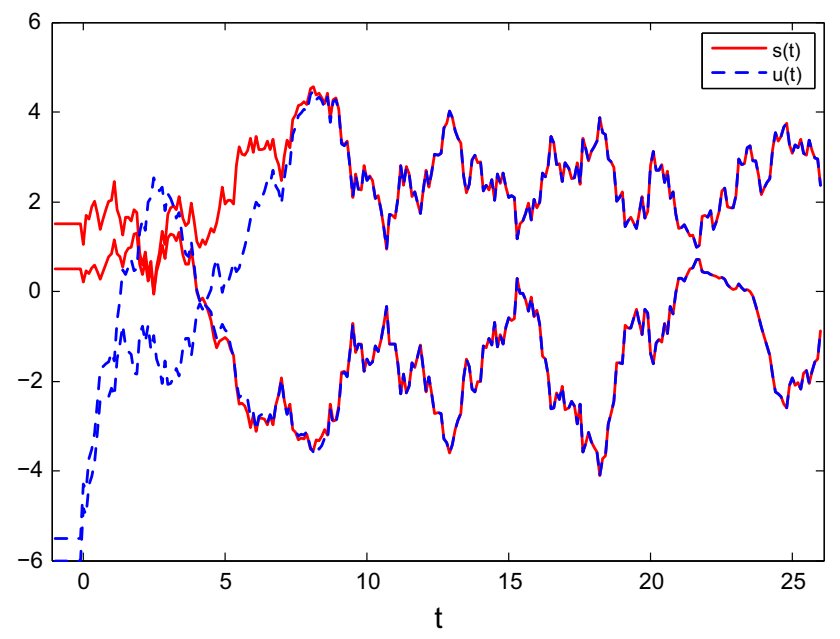

(a)

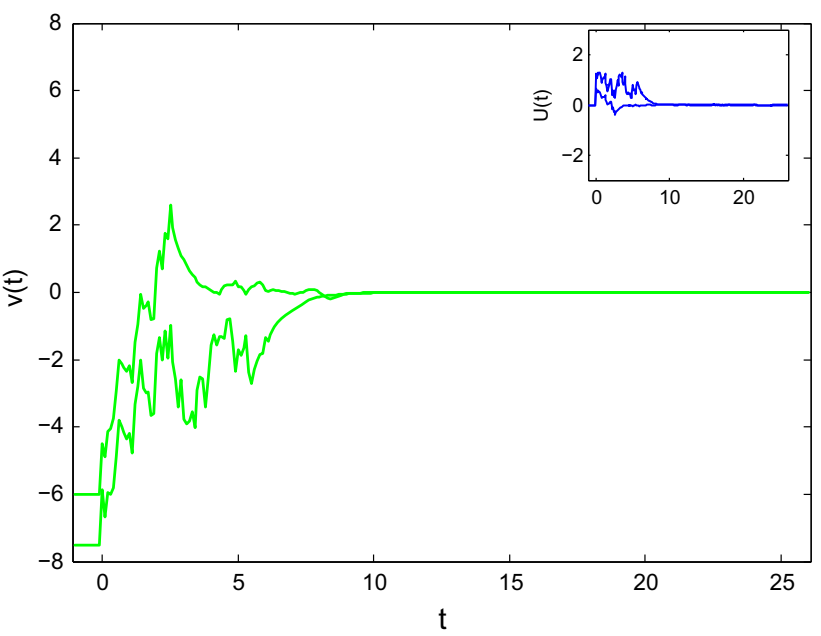

(b)

Fig. 3 Time trajectory of the controlled system (node 6) in Example 1. a The drive signal (red line) and response signal (blue line). b The error signal (green line) and control input (blue line)

Remark 5 Examples 1 and 2 illustrate the effectiveness of the obtained results for SCDNs with different types of time delays, network topologies and reaction-diffusion terms. From the contrast of the uncontrolled and controlled systems in Figs. 2, 3 and 4, we see that adaptive controller is a practical tool to synchronize the networks.

\section{Application to image encryption}

In this section, we will apply the adaptive synchronization of a 2-D SCDN to image encryption based on the spatiotemporal cryptosystem proposed in [4]. The parameters of the drive-response system are taken as follows: $\mathbb{M}=\{1\}, \quad \mathbb{O}=[-10,10], \quad N=1, \quad \tilde{\boldsymbol{D}}=10^{-4} \boldsymbol{I}, \quad \tilde{\boldsymbol{A}}=\boldsymbol{I}$, $\tilde{\boldsymbol{B}}_{3}=\boldsymbol{0}, \quad \boldsymbol{I}(t)=\boldsymbol{0}, \tilde{\boldsymbol{f}}(\varsigma)=\tanh (\varsigma), \tau(t)=\frac{e^{t}}{1+e^{t}}, \quad \zeta(t)=0$, $\tilde{\boldsymbol{g}}\left(\varsigma_{1}, \varsigma_{2}, \varsigma_{3}\right)=\varsigma_{1}$,

$\tilde{\boldsymbol{B}}_{1}=\left[\begin{array}{cc}2 & -0.1 \\ -5 & 4.5\end{array}\right], \quad \tilde{\boldsymbol{B}}_{2}=\left[\begin{array}{cc}-1.5 & -0.1 \\ -0.2 & -4\end{array}\right]$.

The initial conditions are chosen as $s_{1}(\theta, x)=\sin ^{2}\left(\frac{x \pi}{2}\right), s_{2}(\theta, x)=\cos ^{2}\left(\frac{x \pi}{2}\right), x \in \mathbb{O}$,

and $u_{1}(\theta, x)=1, x \in \mathbb{O}_{k}, u_{2}(\theta, x)=-1, x \in C_{\mathbb{O}} \mathbb{O}_{k}$, 


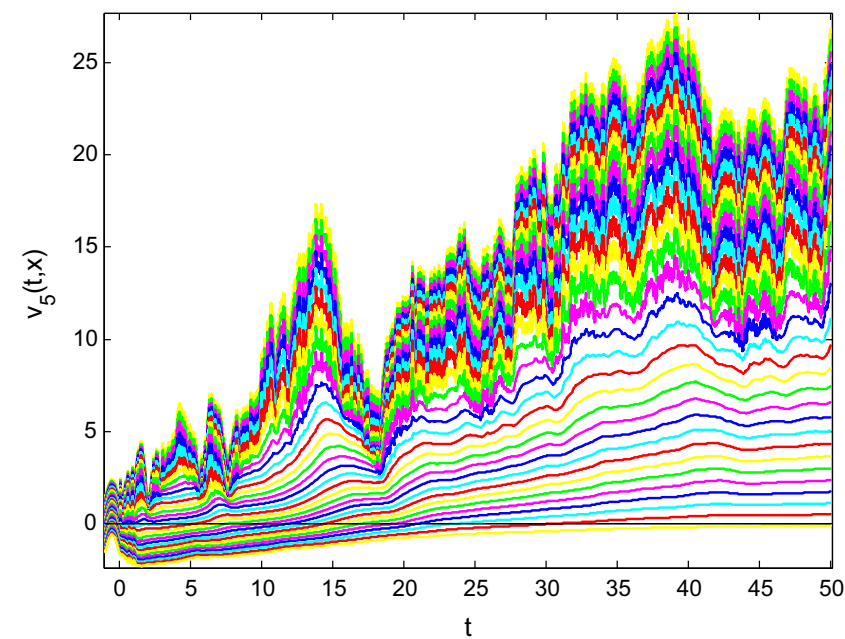

(a)

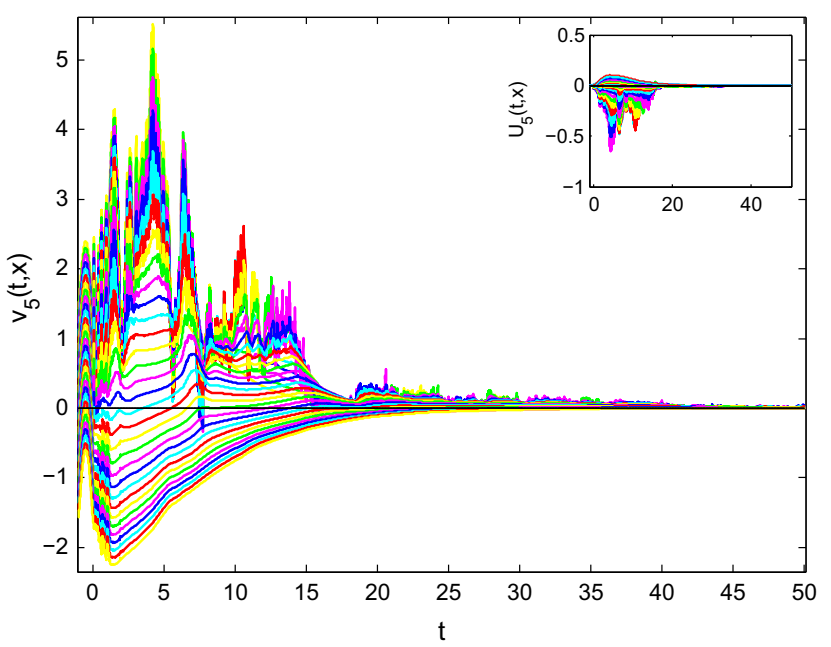

(b)

Fig. 4 The error signals of uncontrolled (left) and controlled system (right) in Example 2

where $-1 \leq \theta \leq 0$ and $\mathbb{O}_{k}=[-10+0.2 k,-9.9+0.2 k]$ $(k \in \mathbb{N}), \quad$ otherwise, $\quad s_{1}(\theta, x)=s_{2}(\theta, x)=u_{1}(\theta, x)$ $=u_{2}(\theta, x)=0$. The dynamical behavior of the uncontrolled error system can be found in Fig. 5, which is obviously unstable. Then, the adaptive control input (7) is added into the error system. According to Corollary 1, the controlled system is synchronous as shown in Fig. 6.

Next, we apply the adaptive synchronization results derived above to image cryptosystem proposed in [4], whose flowchart is shown in Fig. 7. We replace the drive system and response system by the aforementioned 2-D SCDN. To demonstrate the security and effectiveness, we use four different images including Lena (gray $512 \times 512$ ), Lady (color $256 \times 256$ ), Pepper (color $512 \times 512$ ), and SanDiego (color $1024 \times 1024$ ), as shown in Fig. 8. Then, the cryptosystem is evaluated by key space analysis and statistical analysis as follows.

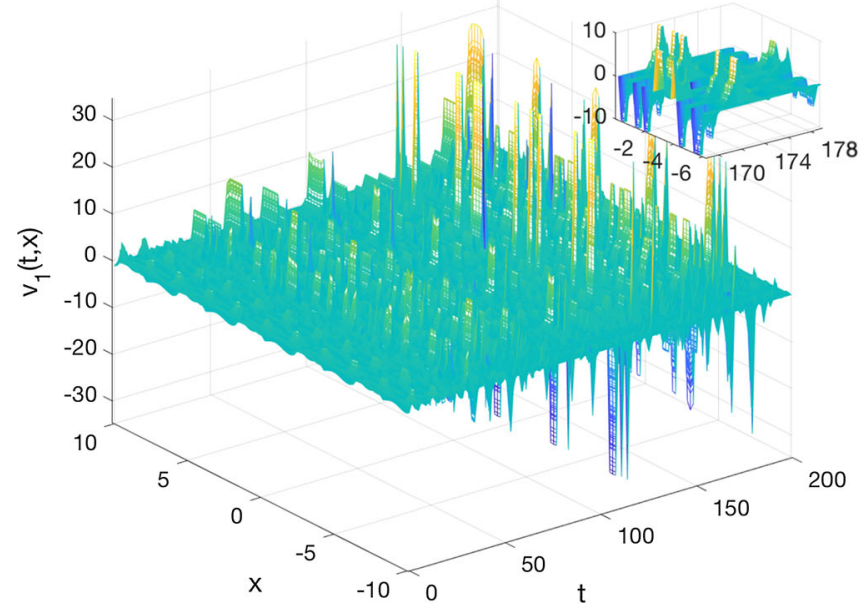

Fig. 5 The error signals of uncontrolled system in Sect. 5

\subsection{Key space analysis}

The used encryption algorithm includes the following keys: the parameters of the spatiotemporal chaotic neural networks, the sampling spatial points, and synchronous time. As stated in [4], the key space is large enough in theory to resist the brute-force attack. In addition, the drive-response system considered here is stochastic chaotic system. The stochastic Brownian motion $\boldsymbol{w}(t)$ can also be treated as the secret key, which is irreproducible. Thus, the uncertainty of Brownian motion has also improved the security of the encryption algorithm.

\subsection{Statistical analysis}

To demonstrate the robustness of cryptosystem, we perform analysis of histogram, mean square error, peak signal-

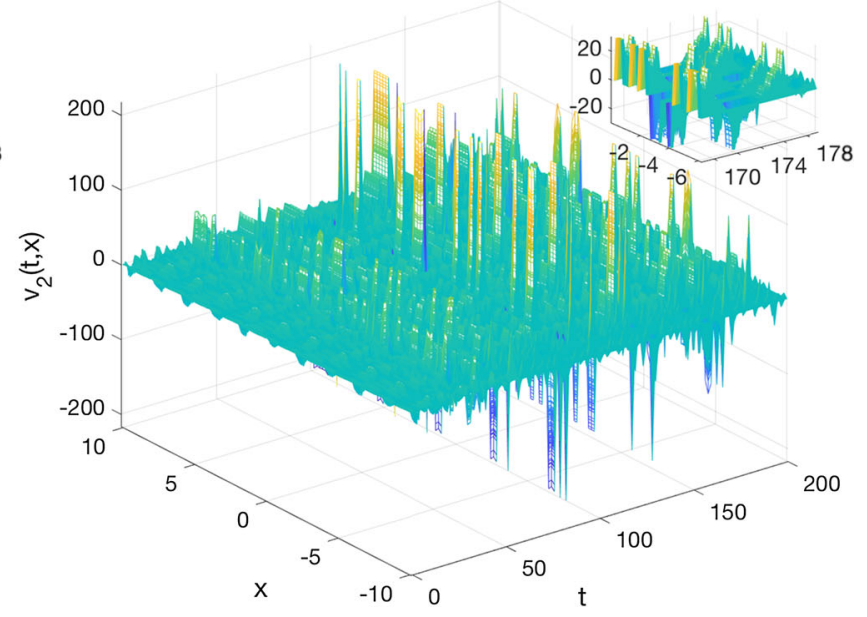



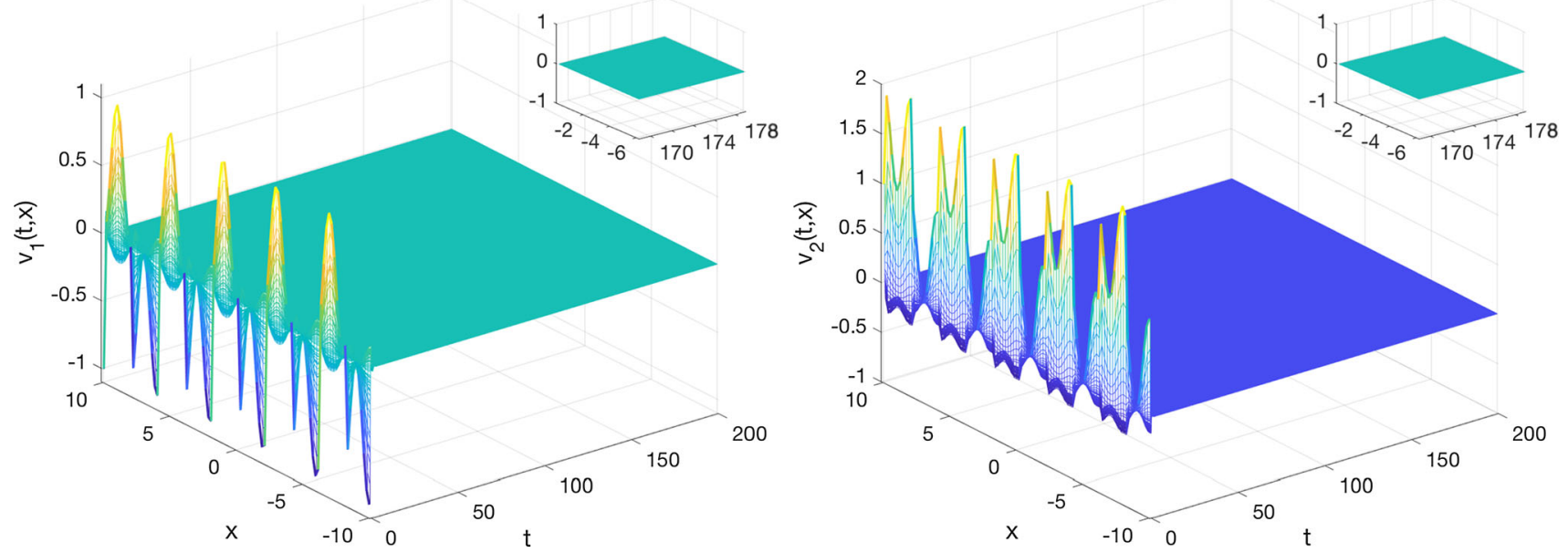

Fig. 6 The error signals of controlled system in Sect. 5

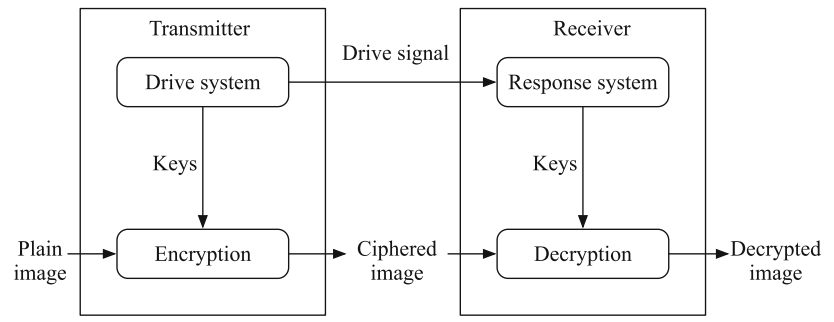

Fig. 7 Flowchart of chaotic secure communication system based on adaptive synchronization

to-noise ratio, entropy, and correlation of the plain images and ciphered images. The ciphered images should process certain random properties, which means the decrease in variance of histogram, the low peak signal-to-noise ratio, closeness of entropy to 8 , and small correlation of plain images and ciphered images and of two adjacent pixels in ciphered images. Figures 9 and 10 illustrate the plain images, ciphered images, decrypted images, and the corresponding histograms. It can be observed that the ciphered images are quite different from the plain images whereas the decrypted images are identical with the plain images. Figures 11, 12 and 13 depict the correlation distribution of two adjacent pixels in horizontal, vertical, and diagonal directions. The significant reduction in correlation indicates that the correlation of adjacent pixels in the plain images has been removed.
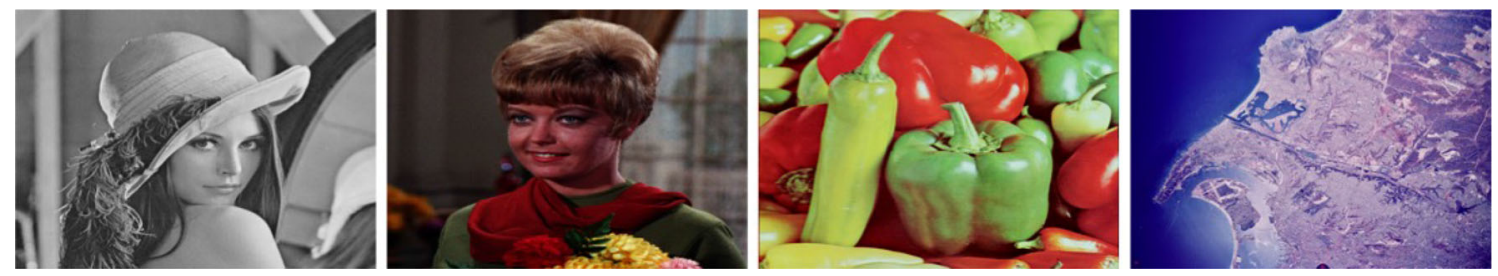

The quantification of the ciphered results is presented in Table 1, 2 and 3, including variance of histogram [42], mean square error (MSE), peak signal-to-noise ratio (PSNR) [9], entropy, and correlation of the plain images and ciphered images (CPC), and correlations of two adjacent pixels [4]. From Figs. 9, 10, and Table 1, we see that the variances of histograms of ciphered images significantly decrease compared with those of plain images. The uniformity of the histograms of the ciphered images makes statistic attacks difficult. As a result, the proposed method is able to resist chosen-plaintext or known-plaintext attacks. From Table 2, the average PSNR 7.96 of Lady, Pepper, SanDiego is smaller than the value 7.99 in [9] and the entropy 7.9993 of Lena is closer to 8 than value 7.9941 in [4]. The correlations of two adjacent pixels in Table 3 corresponds to the description of Figs. 11, 12 and 13, which also implies the random-like appearances of the ciphered images.

The complexity of the used encryption scheme is performed compared with schemes in [4] and [9]. The timeconsuming part here lies in the construction of spatiotemporal chaotic sequences generated by the above SCDN. The time complexity is $\Theta(4 \times M \times N)$, which is same as Chen's algorithm [4], because we exactly use Chen's frame. The main difference is that the Brownian motion is pre-generated before construction of chaotic sequences,

Fig. 8 Four standard images for encryption process 

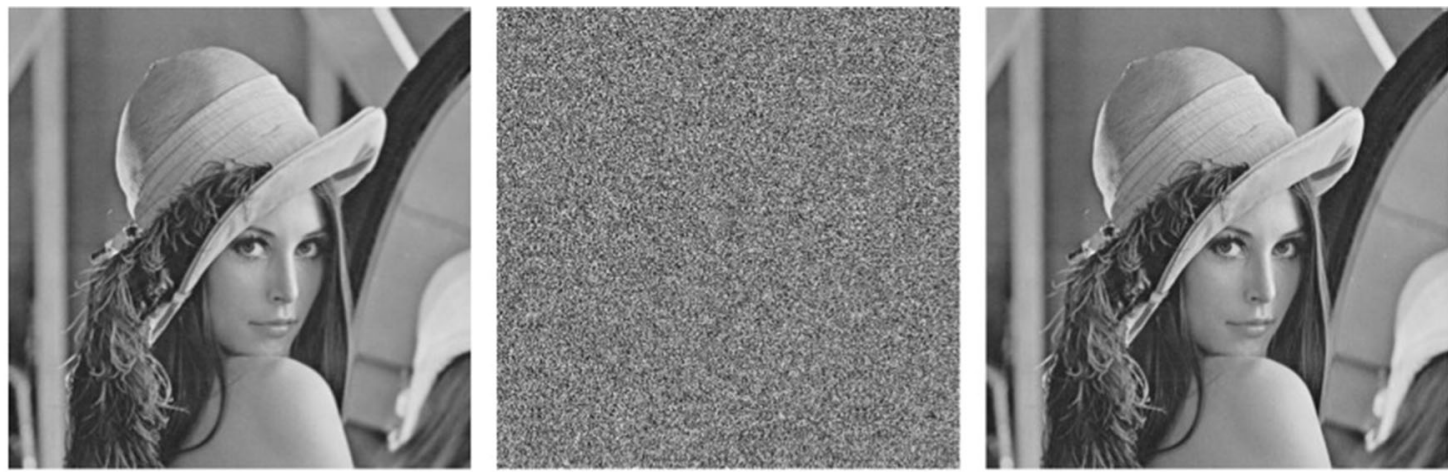

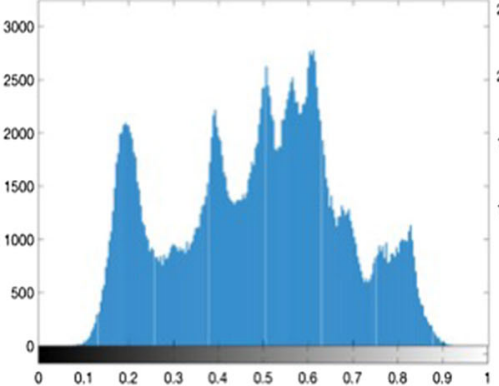

(a)

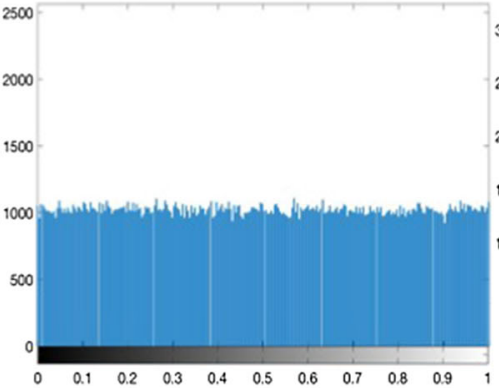

(b)

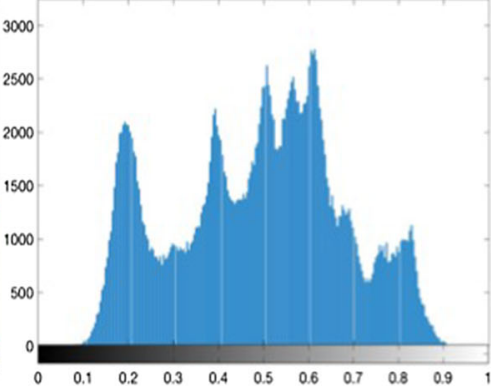

(c)

Fig. 9 Images (top) and corresponding histograms (bottom). a Lena image. b Ciphered image. c Decrypted image
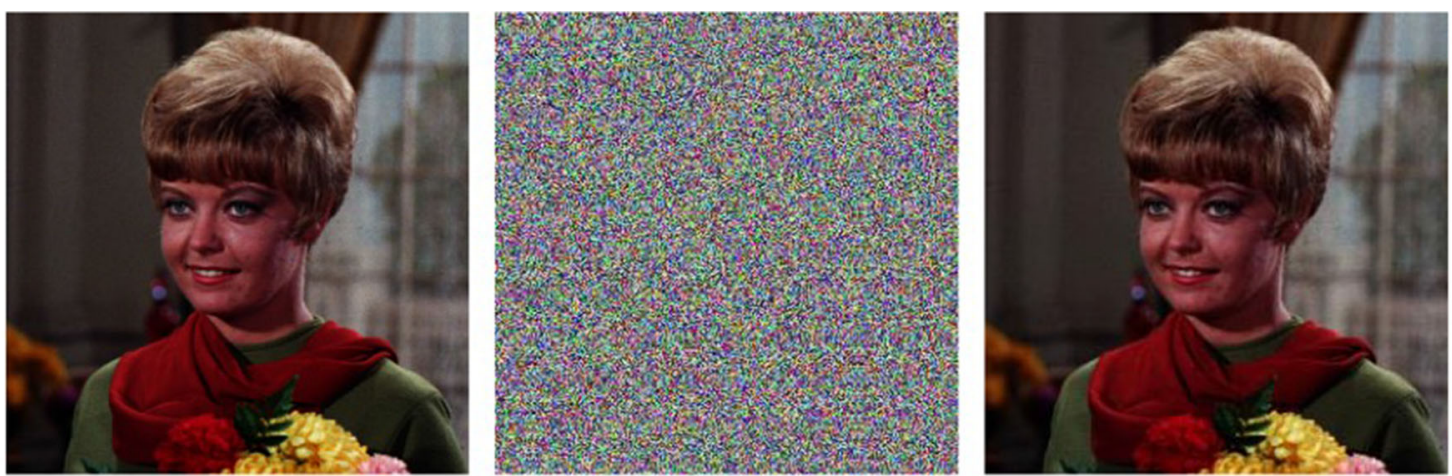

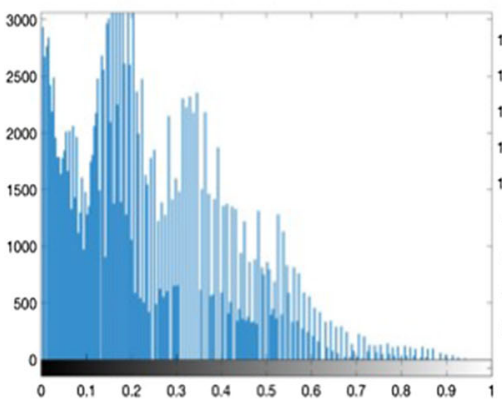

(a)

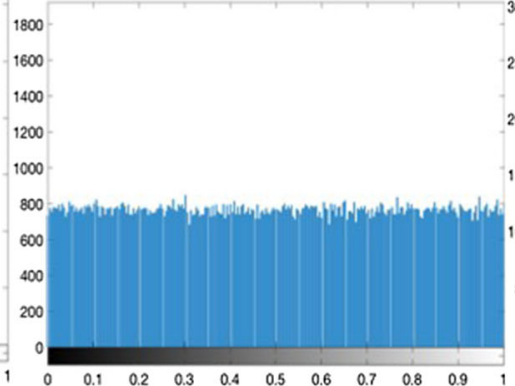

(b)

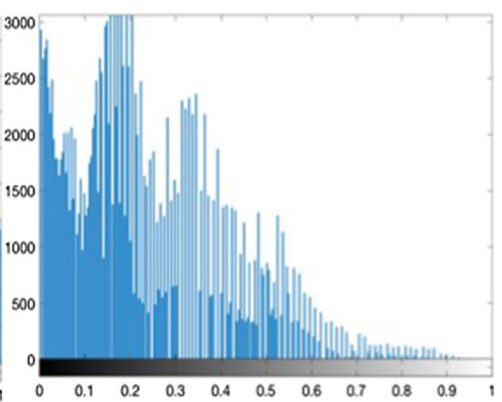

(c)

Fig. 10 Images (top) and corresponding histograms (bottom). a Lady image. b Ciphered image. c Decrypted image

which hardly causes extra time consume. In contrast to Lakshmanan's scheme [9], the used scheme has lower computational cost, because the scheme only depends on the diffusion operation. But implementing the permutation operation in encryption scheme may improve the performance and may not cause significant computational cost. Hence, the future work of encryption scheme will focus on the permutation operation. 

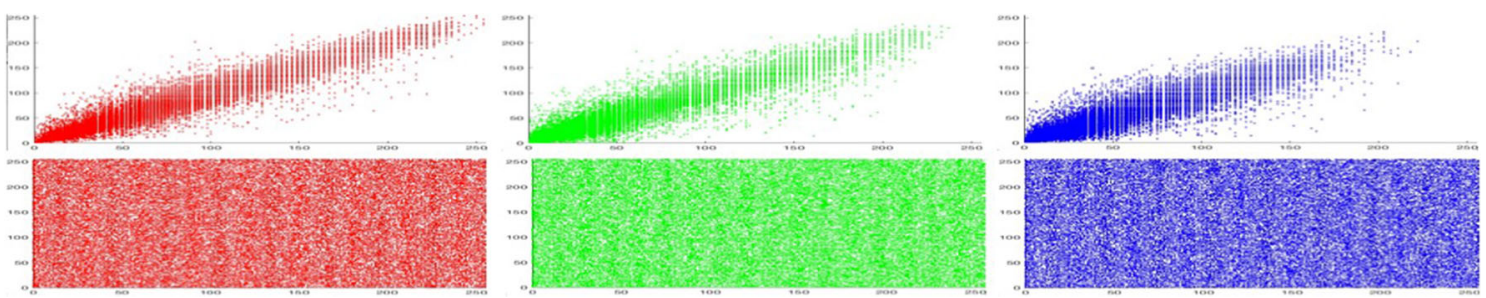

Fig. 11 Correlation of adjacent pixels in plain image (top) and ciphered image (bottom) in horizontal direction
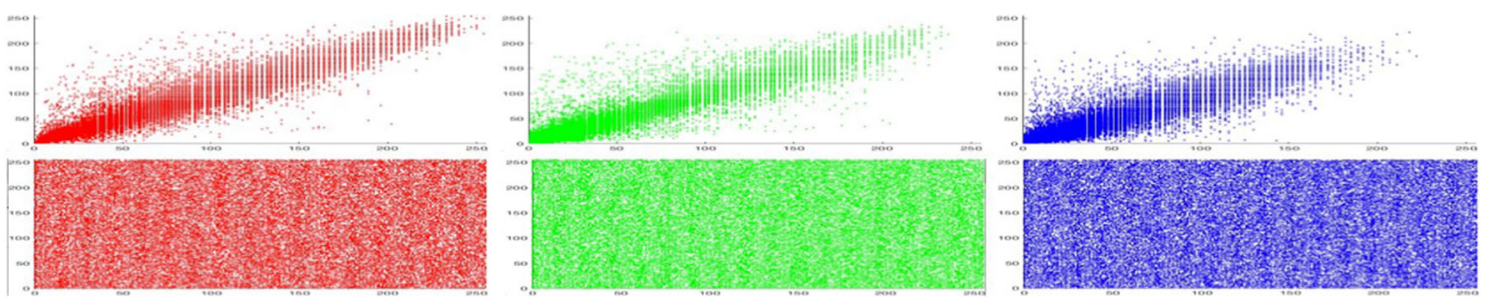

Fig. 12 Correlation of adjacent pixels in plain image (top) and ciphered image (bottom) in vertical direction
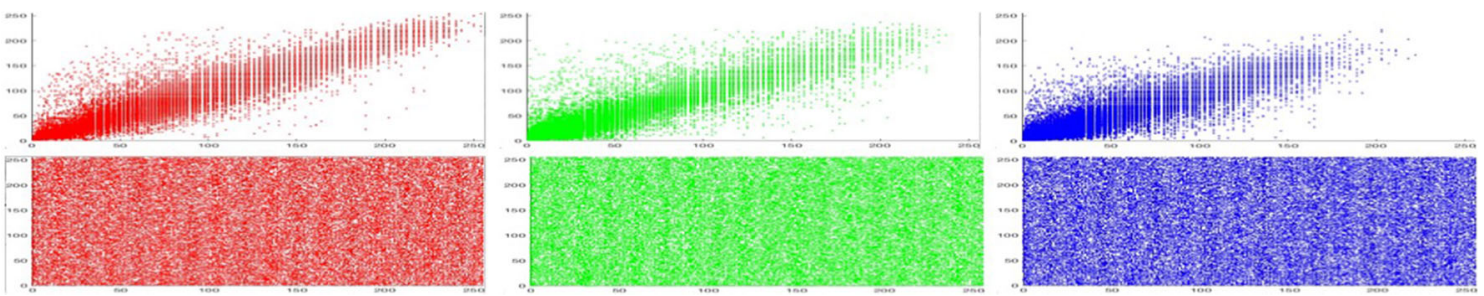

Fig. 13 Correlation of adjacent pixels in plain image (top) and ciphered image (bottom) in diagonal direction

Table 1 Variance of histogram

\begin{tabular}{lllll}
\hline & Lena & Lady & Pepper & SanDiego \\
\hline Original & $6.3701 \mathrm{e} 5$ & $9.0885 \mathrm{e} 5$ & $5.3031 \mathrm{e} 6$ & $9.3502 \mathrm{e} 7$ \\
Ciphered & 987.7969 & 698.625 & $2.6876 \mathrm{e} 3$ & $1.2082 \mathrm{e} 4$ \\
Decrypted & $6.3701 \mathrm{e} 5$ & $9.0885 \mathrm{e} 5$ & $5.3031 \mathrm{e} 6$ & $9.3502 \mathrm{e} 7$ \\
\hline
\end{tabular}

Table 2 Comparison of MSE, PSNR, ENTROPY and CPC of different images

\begin{tabular}{lrrlr}
\hline Image & \multicolumn{1}{c}{ MSE } & PSNR & ENTROPY & \multicolumn{1}{l}{ CPC } \\
\hline Lena & 7861.4 & 9.1758 & 7.9993 & -0.0035 \\
Lady & 12236.7 & 7.2914 & 7.9973 & 0.0014 \\
Pepper & 10198.5 & 8.0956 & 7.9993 & 0.0038 \\
SanDiego & 9282.5 & 8.4955 & 7.9998 & 0.00047 \\
\hline
\end{tabular}

\section{Conclusion}

In this paper, we develop some criteria of exponential synchronization for SCDNs with reaction-diffusion terms and S-type distributed delays. First, we introduce a generalized Halanay inequality to handle the S-type distributed delays. Then, adaptive control and adaptive pinning control
Table 3 Correlations of two adjacent pixels in horizontal, vertical, and diagonal directions

\begin{tabular}{llllrl}
\hline Image & & Horizontal & Vertical & Diagonal & Average \\
\hline Lena & Original & 0.9726 & 0.9863 & 0.9596 & 0.9728 \\
& Ciphered & 0.0044 & 0.0028 & -0.0016 & 0.0029 \\
Lady & Original & 0.9677 & 0.9596 & 0.9453 & 0.9575 \\
& Ciphered & 0.0064 & 0.0030 & 0.0028 & 0.0041 \\
\multirow{2}{*}{ Pepper } & Original & 0.9704 & 0.9715 & 0.9576 & 0.9665 \\
& Ciphered & 0.0028 & 0.0040 & 0.0011 & 0.0026 \\
\multirow{2}{*}{ SanDiego } & Original & 0.9173 & 0.9143 & 0.8946 & 0.9087 \\
& Ciphered & 0.00082 & 0.00065 & 0.00052 & 0.00066 \\
\hline
\end{tabular}

strategies of exponential synchronization are established by utilizing Poincaré inequality and constructing simple Lyapunov-Krasovskii functional candidate without the distributed terms. Finally, we present two numerical examples and apply a 2-D SCDN to a spatiotemporal cryptosystem proposed in [4]. Experimental results, including decrease in variance of histogram decreases, low PSNR, closeness of entropy to 8, and small correlation between plain images and ciphered images, show effective application of the obtained theoretical results. The future 
work will focus on implementing the permutation operation in encryption scheme, since NPCR (number of pixels change rate) and UACI (unified average changing intensity) cannot reach desired performance and the permutation operation hopefully overcomes the issues.

Acknowledgements The authors would like to thank the editor, associate editor, and reviewers for their constructive comments and fruitful suggestions, which greatly improved this manuscript. The work was partly supported by National Natural Science Foundation of China (No. 11771014), Fundamental Research Funds for Central Universities (No. 06500073), and China Scholarship Council (File No. 201706330011).

Open Access This article is distributed under the terms of the Creative Commons Attribution 4.0 International License (http://creative commons.org/licenses/by/4.0/), which permits unrestricted use, distribution, and reproduction in any medium, provided you give appropriate credit to the original author(s) and the source, provide a link to the Creative Commons license, and indicate if changes were made.

\section{References}

1. Ahmed MAA, Liu Y, Zhang W, Alsaadi FE (2017) Exponential synchronization via pinning adaptive control for complex networks of networks with time delays. Neurocomputing 225:198-204

2. Ali MS, Yogambigai J (2017) Finite-time robust stochastic synchronization of uncertain markovian complex dynamical networks with mixed time-varying delays and reaction-diffusion terms via impulsive control. J Franklin Inst 354(5):2415-2436

3. Arenas A, Díaz-Guilera A, Pérez-Vicente CJ (2006) Synchronization reveals topological scales in complex networks. Phys Rev Lett 96(11):114,102

4. Chen WH, Luo S, Zheng WX (2016) Impulsive synchronization of reaction-diffusion neural networks with mixed delays and its application to image encryption. IEEE Trans Neural Netw Learn Syst 27(12):2696-2710

5. Chen X, Zhao Z, Song Q, Hu J (2017) Multistability of complexvalued neural networks with time-varying delays. Appl Math Comput 294:18-35

6. Halanay A (1966) Differential equations: stability, oscillations, time lags. Academic Press, New York

7. Kao Y, Gao C, Han W (2010) Global exponential robust stability of reaction-diffusion interval neural networks with continuously distributed delays. Neural Comput Appl 19(6):867-873

8. Kwon OM, Lee SM, Park JH (2010) Improved delay-dependent exponential stability for uncertain stochastic neural networks with time-varying delays. Phys Lett A 374(10):1232-1241

9. Lakshmanan S, Prakash M, Lim CP, Rakkiyappan R, Balasubramaniam P, Nahavandi S (2018) Synchronization of an inertial neural network with time-varying delays and its application to secure communication. IEEE Trans Neural Netw Learn Syst 29(1):195-207

10. Lee TH, Park JH, Jung HY, Lee SM, Kwon OM (2012) Synchronization of a delayed complex dynamical network with free coupling matrix. Nonlinear Dyn 69(3):1081-1090

11. Li XJ, Yang GH (2016) Fls-based adaptive synchronization control of complex dynamical networks with nonlinear couplings and state-dependent uncertainties. IEEE Trans Cybern 46(1):171-180
12. Liang J, Gong W, Huang T (2016) Multistability of complexvalued neural networks with discontinuous activation functions. Neural Netw 84:125-142

13. Liang X, Wang L, Wang Y, Wang R (2016) Dynamical behavior of delayed reaction-diffusion hopfield neural networks driven by infinite dimensional wiener processes. IEEE Trans Neural Netw Learn Syst 27(9):1816-1826

14. Liu W, Guan ZH (2011) Global exponential synchronization of stochastic switching networks with time-varying delay. Asian J Control 13(6):893-902

15. Pastor-Satorras R, Rubi M, Diaz-Guilera A (2003) Statistical mechanics of complex networks. Springer, Berlin

16. Qin J, Gao H, Zheng WX (2015) Exponential synchronization of complex networks of linear systems and nonlinear oscillators: a unified analysis. IEEE Trans Neural Netw Learn Syst 26(3):510-521

17. Rakkiyappan R, Sakthivel N, Cao J (2015) Stochastic sampleddata control for synchronization of complex dynamical networks with control packet loss and additive time-varying delays. Neural Netw 66:46-63

18. Saravanakumar R, Ali MS, Karimi HR (2017) Robust $h_{\infty}$ control of uncertain stochastic markovian jump systems with mixed timevarying delays. Int J Syst Sci 48(4):862-872

19. Senana S, Ali MS, Vadivel R, Arik S (2017) Decentralized eventtriggered synchronization of uncertain markovian jumping neutral-type neural networks with mixed delays. Neural Netw 86:32-41

20. Sheikhan M, Shahnazi R, Garoucy S (2013) Synchronization of general chaotic systems using neural controllers with application to secure communication. Neural Comput Appl 22(2):361-373

21. Steura E, Tyukinb I, Nijmeijera H (2009) Semi-passivity and synchronization of diffusively coupled neuronal oscillators. Physica D 238(21):2119-2128

22. Strogatz SH (2001) Exploring complex networks. Nature 410:268-276

23. Temam R (2012) Infinite-dimensional dynamical systems in mechanics and physics. Springer, Berlin

24. Uhlhaas PJ, Singer W (2006) Neural synchrony in brain disorders: relevance for cognitive dysfunctions and pathophysiology. Neuron 52(1): 155-168

25. Wang L (2016) Global well-posedness and stability of the mild solutions for a class of stochastic partial functional differential equations. Sci China Math 47:371-382

26. Wang L, Wang Y (2011) Global exponential stabilization for a class of distributed parameter control systems with Markovian jumping parameters and time-varying delay. J Vib Control 17(6):873-880

27. Wang L, Xu D (2003) Global exponential stability of hopfield reaction-diffusion neural networks with time-varying delays. Sci China Inform Sci 46(6):466-474

28. Wang L, Zhang R, Wang Y (2009) Global exponential stability of reaction-diffusion cellular neural networks with s-type distributed time delays. Nonlinear Anal RWA 10(2):1101-1113

29. Wang L, Zhao H (2014) Synchronized stability in a reactiondiffusion neural network model. Phys Lett A 378(48):3586-3599

30. Wang W, Li L, Peng H, Xiao J, Yang Y (2014) Stochastic synchronization of complex network via a novel adaptive nonlinear controller. Nonlinear Dyn 76(1):591-598

31. Wang Z, Liu Y, Yu L, Liu X (2006) Exponential stability of delayed recurrent neural networks with Markovian jumping parameters. Phys Lett A 356(4):346-352

32. Wei T, Wang L, Wang Y (2017) Existence, uniqueness and stability of mild solutions to stochastic reaction-diffusion CohenGrossberg neural networks with delays and wiener processes. Neurocomputing 239:19-27 
33. Wen S, Zeng Z, Huang T, Meng Q, Yao W (2015) Lag synchronization of switched neural networks via neural activation function and applications in image encryption. IEEE Trans Neural Netw Learn Syst 26(7):1493-1502

34. Wu ZG, Park JH, Su H, Song B, Chu J (2013) Exponential synchronization for complex dynamical networks with sampleddata. SIAM J Control Optim 51(1):3486-3510

35. Wu ZG, Shi P, Su H, Chu J (2013) Stochastic synchronization of markovian jump neural networks with time-varying delay using sampled data. IEEE Trans Cybern 43(6):1796-1806

36. Xu D, Wang X, Yang Z (2013) Further results on existenceuniqueness for stochastic functional differential equations. Sci China Math 56(6):1169-1180

37. Yang X, Cao J, Yang Z (2013) Synchronization of coupled reaction-diffusion neural networks with time-varying delays via pinning-impulsive controller. SIAM J Control Optim 51(5):3486-3510

38. Yu W, Chen G, Lü J, Kurths J (2013) Synchronization via pinning control on general complex networks. SIAM J Control Optim 51(2):1395-1416

39. Zhang Q, Lu J, Lü J, Tse CK (2008) Adaptive feedback synchronization of a general complex dynamical network with delayed nodes. IEEE Trans Circuits Syst II 55(2):183-187

40. Zhang YQ, He Y, Wang XY (2018) Spatiotemporal chaos in mixed linear-nonlinear two-dimensional coupled logistic map lattice. Physica A 490:148-160
41. Zhang YQ, Wang XY (2014) Spatiotemporal chaos in mixed linear-nonlinear coupled logistic map lattice. Physica A 402:104-118

42. Zhang YQ, Wang XY (2014) A symmetric image encryption algorithm based on mixed linear-nonlinear coupled map lattice. Inf Sci 273:329-351

43. Zhang YQ, Wang XY (2015) A new image encryption algorithm based on non-adjacent coupled map lattices. Appl Soft Comput 26:10-20

44. Zhang YQ, Wang XY, Liu J, Chi ZL (2016) An image encryption scheme based on the MLNCML system using DNA sequences. Opt Lasers Eng 82:95-103

45. Zhang YQ, Wang XY, Liu LY, He Y, Liu J (2017) Spatiotemporal chaos of fractional order logistic equation in nonlinear coupled lattices. Commun Nonlinear Sci Numer Simul 52:52-61

46. Zhao DW, Wang LH, Zhi YF, Zhang J, Wang Z (2015) The robustness of multiplex networks under layer node-based attack. Sci Rep 6:24,304

47. Zhou J, Lu J, Lü J (2008) Pinning adaptive synchronization of a general complex dynamical network. Automatica 44:996-1003

48. Zhu Q, Cao J (2011) Exponential stability of stochastic neural networks with both Markovian jump parameters and mixed time delays. IEEE Trans Syst Man Cybern Part B 41(2):341-353 